Absolute continuity for operator valued completely positive maps on $C^{*}$-algebras Aurelian Gheondea, and Ali Şamil Kavruk

Citation: Journal of Mathematical Physics 50, 022102 (2009);

View online: https://doi.org/10.1063/1.3072683

View Table of Contents: http://aip.scitation.org/toc/jmp/50/2

Published by the American Institute of Physics

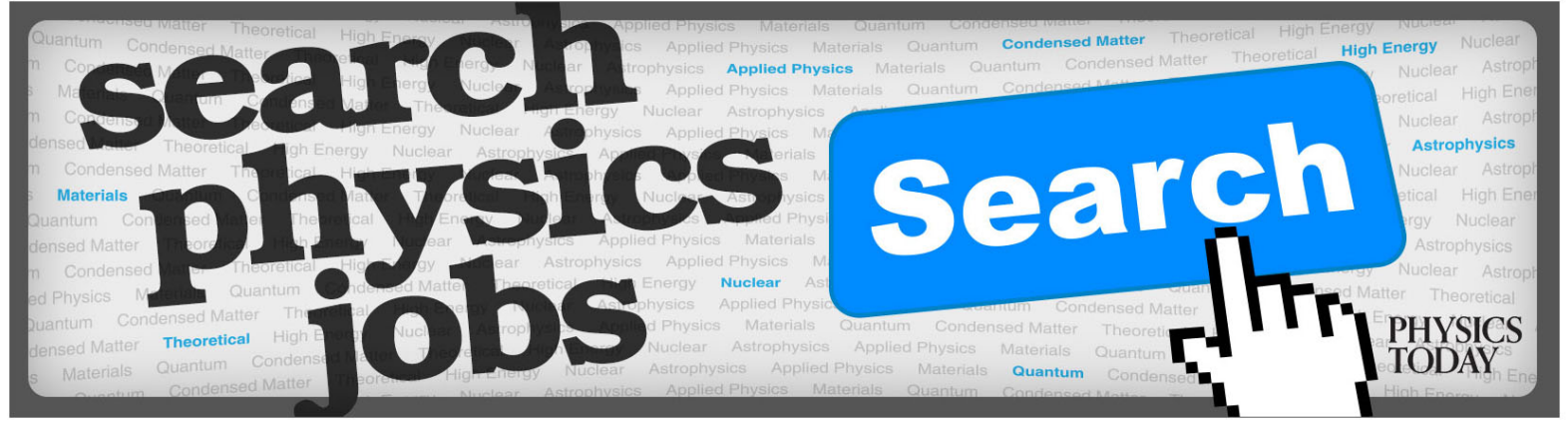




\title{
Absolute continuity for operator valued completely positive maps on $C^{*}$-algebras
}

\author{
Aurelian Gheondea ${ }^{1, a)}$ and Ali Şamil Kavruk ${ }^{2, b)}$ \\ ${ }^{1}$ Department of Mathematics, Bilkent University, Bilkent, Ankara 06800, Turkey \\ and Institutul de Matematică al Academiei Române, C. P. 1-764, 014700 Bucureşti, \\ Romania \\ ${ }^{2}$ Department of Mathematics, University of Houston, Houston, Texas 77204-3476, USA
}

(Received 15 October 2008; accepted 16 December 2008; published online 11 February 2009)

\begin{abstract}
Motivated by applicability to quantum operations, quantum information, and quantum probability, we investigate the notion of absolute continuity for operator valued completely positive maps on $C^{*}$-algebras, previously introduced by Parthasarathy [in Athens Conference on Applied Probability and Time Series Analysis I (SpringerVerlag, Berlin, 1996), pp. 34-54]. We obtain an intrinsic definition of absolute continuity, we show that the Lebesgue decomposition defined by Parthasarathy is the maximal one among all other Lebesgue-type decompositions and that this maximal Lebesgue decomposition does not depend on the jointly dominating completely positive map, we obtain more flexible formulas for calculating the maximal Lebesgue decomposition, and we point out the nonuniqueness of the Lebesgue decomposition as well as a sufficient condition for uniqueness. In addition, we consider Radon-Nikodym derivatives for absolutely continuous completely positive maps that, in general, are unbounded positive self-adjoint operators affiliated to a certain von Neumann algebra, and we obtain a spectral approximation by bounded Radon-Nikodym derivatives. An application to the existence of the infimum of two completely positive maps is indicated, and formulas in terms of Choi's matrices for the Lebesgue decomposition of completely positive maps in matrix algebras are obtained. (C) 2009 American Institute of Physics. [DOI: 10.1063/1.3072683]
\end{abstract}

\section{INTRODUCTION}

Modern quantum theory intensively uses the notion of operator valued completely positive maps on $C^{*}$-algebras as a mathematical model for quantum operations (e.g., see, Davies ${ }^{12}$ and Kraus $\left.^{24}\right)$. In quantum probability, operator valued completely positive maps on $C^{*}$-algebras play the role of transition probability mappings for quantum Markov processes (e.g., see Meyer ${ }^{26}$ and Parthasarthy ${ }^{27}$ ). An essential role in the comparison of operator valued completely positive maps on $C^{*}$-algebras is played by the Radon-Nikodym derivative for completely positive maps, first introduced and studied by Arveson. ${ }^{6}$ Slightly more general Radon-Nikodym derivatives have been considered by Belavkin and Stazsewski, ${ }^{9}$ while Raginsky ${ }^{31}$ illustrated the importance of these results and related ideas to some special problems in quantum information theory, and Belavkin et $a l .{ }^{8}$ applied this Radon-Nikodym derivative to define and investigate a minimax fidelity for quantum channels.

From the point of view of quantum measurements, the natural setting is that of open quantum systems for which admissible devices are modeled by quantum operations, that is, completely positive maps on the $C^{*}$-algebra of observables of the physical system. The cone of completely

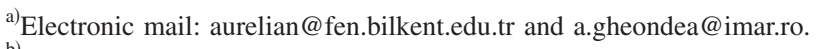

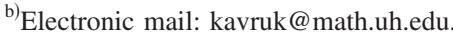


positive maps defines a natural partial order relation. The main tool to deal with completely positive maps is the minimal Stinespring representation that produces a "larger" Hilbert space $\mathcal{K}$ and a representation $\pi$ of the $C^{*}$-algebra $\mathcal{A}$ of observables on $\mathcal{K}$.

An intrinsic deficiency of the Radon-Nikodym derivative is the requirement that the two completely positive maps should be somehow comparable, otherwise the Radon-Nikodym derivative may not exist. Recalling the classical Lebesgue decomposition, which represents a measure $\mu$ as the superposition of the "good" (absolutely continuous) part and the "bad" (singular) part, with respect to another measure $\nu$, the next step in the comparison theory for operator valued completely positive maps on $C^{*}$-algebras was naturally pointing toward investigations on Lebesguetype decompositions. This requires first the clarification of absolute continuity and singularity in this noncommutative setting. Motivated by quantum probability, this further step was done by Parthasarthy ${ }^{28}$ who exhibited, for two unital completely positive maps $\varphi, \psi: \mathcal{A} \rightarrow \mathcal{B}(\mathcal{H})$, where $\mathcal{A}$ is a $C^{*}$-algebras and $\mathcal{H}$ is a Hilbert space, a construction inspired by the classical Lebesgue decomposition theorem that can be used in order to produce a decomposition $\varphi=\varphi_{\mathrm{ac}}+\varphi_{\mathrm{s}}$, where $\varphi_{\mathrm{ac}}$ and $\varphi_{\mathrm{s}}$ are again completely positive and they are called, respectively, the absolutely continuous part and, respectively, the singular part, of $\varphi$ with respect to $\psi$. Consequently, $\varphi$ is called $\psi$-absolutely continuous if $\varphi_{\mathrm{s}}=0$. However, natural questions on intrinsic characterizations of absolute continuity and singularity, uniqueness of Lebesgue decompositions, as well as more flexible formulas for calculating them are left unanswered.

Further, Radon-Nikodym derivative as a bounded operator can be obtained only when comparable completely positive maps are used, while for relatively absolutely continuous completely positive maps that are not comparable (with respect to the natural order relation) the RadonNikodym derivative can be defined as an unbounded operator only. This raises other questions on approximation properties, e.g., in the spectral sense, of this unbounded Radon-Nikodym derivative.

Our approach to the aforementioned questions, from the perspective of quantum measurements theory, is based on the observation that the minimal Stinespring representation of a quantum operation $\theta$ is a dilation to a larger ambient Hilbert space $\mathcal{K}$, in which Radon-Nikodym derivatives can be considered as a transcription of the information carried by the quantum operations dominated by $\theta$ to quantum effects mathematically modeled by contractive positive operators on $\mathcal{K}$. Thus, given two operator valued completely positive maps $\varphi$ and $\psi$, on the same $C^{*}$-algebra, what we first do is to make the dilation by means of the minimal Stinespring representation corresponding to a completely positive map $\theta$ that dominated both $\varphi$ and $\psi$, and then to find Lebesgue-type decompositions for the corresponding Radon-Nikodym derivatives. In this way, we can generally reduce most of the obstructions to the known theories of Ando in Ref. 3 of Lebesgue decompositions for positive contractions and that of $\operatorname{Simon}^{33}$ on canonical forms for non-negative quadratic forms (Jøorgensen ${ }^{19}$ considered similar canonical forms for unbounded operators), combined with the observations of Kosaki (cf. Refs. 21 and 22) on the connections between these noncommutative Lebesgue-type decompositions, as well as his refinements of the Ando's theory. However, in doing this there is a price we have to pay: the representation is living within a certain von Neumann algebra, the commutant of the representation of the $C^{*}$-algebra on $\mathcal{K}$, which can be rather narrow when compared to the ambient $\mathcal{B}(\mathcal{K})$. This constraint yields a series of additional obstructions especially in connection with uniqueness.

Following this chain of ideas, we actually show that, to a certain extent, the above mentioned results of Ando, Simon, and Kosaki can be streamlined to the case of operator valued completely positive maps on $C^{*}$-algebras in such a way that the construction of the Lebesgue decomposition, as well as the underlying notion of absolute continuity employed by Parthasarthy, fitted into this general framework. Moreover, even though the relations between the existing comparison theories for non-negative bounded operators, for operator valued completely positive maps on $C^{*}$-algebras, and that for non-negative quadratic forms look rather intricate, at least at the first glance, we can show that one can smoothly translate concepts and results between them once the dictionary is available. In this paper the point of view is to place the comparison theory for non-negative bounded operators in the center and derive the connections starting from here, but we also indicate 
that, to a certain extent, the three noncommutative comparison theories, for completely positive maps, for non-negative bounded operators, and for non-negative quadratic forms, are equivalent.

The article is organized as follows. In Sec. II we first briefly review the construction and the main results on the Radon-Nikodym derivatives for completely positive maps which enables us to apply the results within the aforementioned theories of Ando, Kosaki, and Simon. The main ingredient is the minimal Stinespring representation for operator valued completely positive maps on $C^{*}$ algebras. From here, it becomes natural to get the intrinsic definition of absolute continuity for operator valued completely positive maps on $C^{*}$-algebras. Then we reformulate the chain rule in a more natural setting, we extend the Radon-Nikodym derivative to absolutely continuous positive definite maps, and get a spectral approximation of it by bounded Radon-Nikodym derivatives.

The main results are contained in Sec. III in which we first show, see Theorem 3.1, that the Lebesgue-type construction of Parthsarathy in Ref. 28 produces actually the maximal Lebesgue decomposition, such that the maximality property makes it unique, and in which absolute continuity and singularity are exactly the natural concepts considered in Sec. II. Then, in Theorem 3.3, we show that the maximal Lebesgue decomposition does not depend on the jointly dominating completely positive map and, hence, that more flexible formulas become available.

The question on the uniqueness of the Lebesgue decomposition for operator valued completely positive maps is much more difficult than the corresponding results for operators. Some sufficient conditions for uniqueness are considered in Proposition 3.8 but here the results are less complete, at this level of generality, because they require a more detailed investigation of Lebesgue decompositions for positive operators in an ambient von Neumann algebra that may be different of $\mathcal{B}(\mathcal{H})$, and hence, different techniques may be needed. Some of these difficulties, especially in connection with dealing with dense operator ranges relative to von Neumann algebras and a still open problem of Dixmier, are apparent from the investigations of Kosaki in Ref. 22. By considering the recovering of the comparison theory for non-negative operators we reduce the question of providing examples of nonuniqueness to the known results of Ando in Ref. 3, but the general uniqueness problem remains for later investigations.

In Sec. IV we illustrate the applicability of the Radon-Nikodym derivative and Lebesgue-type decomposition to the infimum problem for completely positive maps, cf. Theorem 4.2. Other applications to known special similarity problems for operator valued completely bounded maps on $C^{*}$-algebras will be published elsewhere.

In Sec. $\mathrm{V}$ we specialize in completely positive maps in matrices and indicate how to calculate the Radon-Nikodym derivatives and Lebesgue decompositions in terms of the Choi matrices that might be interesting for applications to quantum information theory, e.g., see Ref. 25. In this finite dimensional setting, a key role is played by the tracial completely positive map that is maximal in a certain sense, cf. Raginsky ${ }^{31}$ and the bibliography cited there.

Appendix A contains a review of the Radon-Nikodym derivative and Lebesgue decompositions for bounded non-negative operators on a Hilbert space, where the most important ingredients are two binary operations called parallel sum and shorted operator. This Appendix, hopefully, will make our article more readable. In this operator setting, the notions of absolute continuity and singularity appear naturally, formulas to calculate the Radon-Nikodym derivative and Lebesgue decompositions are available, and questions like uniqueness of Lebesgue decompositions can be answered in more concrete terms.

Appendix B contains the technicalities related to the extension of the Radon-Nikodym derivatives for two relatively absolutely continuous completely positive maps as well as an attempt to a better understanding of the bad part, that is, singular part (from a different perspective, an even more general theory of Lebesgue decompositions for "linear relations," that offers a better understanding of the singular part, was recently considered in Ref. 18). In this respect, it appears to be quite natural to first consider a comparison theory for non-negative quadratic forms on vector spaces and so we do. Then we show that this contains as a special case the comparison theory for completely bounded maps, and hence, it allows us to establish the connection with certain canonical decompositions investigated previously by $\operatorname{Simon}^{33}$ for quadratic forms and, respectively, by 
Jørgensen ${ }^{19}$ for unbounded operators, and, consequently, we get also a spectral approximation property of the Radon-Nikodym derivatives of two relatively absolutely continuous completely positive maps by bounded Radon-Nikodym derivatives, as in Theorem 2.11.

Finally, in Appendix C, we indicate the interdependencies of the three existing noncommutative comparison theories by showing how the one for bounded positive operators and the one for quadratic forms can be obtained from the comparison theory of operator valued completely positive maps on $C^{*}$-algebras.

Radon-Nikodym derivatives for different types of non-negative forms on either von Neumann algebras or $C^{*}$-algebra have been investigated for a long time and a very general theory for *-algebras has been considered by Gudder ${ }^{16}$ (a good source of literature on this type of questions as well). For the special case of normal positive forms on von Neumann algebras, a comparison theory was performed by Kosaki. ${ }^{22}$ From a certain perspective, these are special cases of operator valued completely positive maps, but we consider this as a different direction of investigation that we do not pursue in this article.

\section{RADON-NIKODYM DERIVATIVES OF COMPLETELY POSITIVE MAPS}

\section{A. Completely positive maps}

Assume $\mathcal{A}$ is a unital $C^{*}$-algebra and let $\mathcal{H}$ be a Hilbert space. A linear mapping $\varphi: \mathcal{A}$ $\rightarrow \mathcal{B}(\mathcal{H})$ is positive if $\varphi\left(\mathcal{A}^{+}\right) \subseteq \mathcal{B}(\mathcal{H})^{+}$, that is, it maps positive elements into positive operators

For $n \in \mathbb{N}$ let $M_{n}$ denote the $C^{*}$-algebra of $n \times n$ complex matrices, identified with the $C^{*}$-algebra $\mathcal{B}\left(\mathrm{C}^{n}\right)$. The $C^{*}$-algebra $\mathcal{A} \otimes M_{n}$ identified with the $C^{*}$-algebra $M_{n}(\mathcal{A})$ of $n \times n$ matrices with entries in $\mathcal{A}$ has natural norm and order relation for self-adjoint elements, induced by the embedding $M_{n}(\mathcal{A}) \subseteq \mathcal{B}\left(\mathcal{H} \otimes \mathrm{C}^{n}\right)=\mathcal{B}\left(\mathcal{H}^{n}\right)$, where $\mathcal{H}^{n}$ denotes the Hilbert space direct sum of $n$ copies of $\mathcal{H}$. Using these considerations, a linear mapping $\varphi: \mathcal{A} \rightarrow \mathcal{B}(\mathcal{H})$ is completely positive if for any $n \in \mathbb{N}$ the mapping $\varphi_{n}=\varphi \otimes I_{n}: \mathcal{A} \otimes M_{n} \rightarrow \mathcal{B}\left(\mathcal{H}^{n}\right)$ is positive. Note that, with respect to the identification $\mathcal{A} \otimes M_{n}=M_{n}(\mathcal{A})$, the mapping $\varphi_{n}$ is given by

$$
\varphi_{n}\left(\left[a_{i j}\right]_{i, j=1}^{n}\right)=\left[\varphi\left(a_{i j}\right)\right]_{i, j=1}^{n}, \quad\left[a_{i j}\right]_{i, j=1}^{n} \in M_{n}(\mathcal{A}) .
$$

A linear map $\varphi: \mathcal{A} \rightarrow \mathcal{B}(\mathcal{H})$ is called positive definite if for all $n \in \mathbb{N},\left(a_{j}\right)_{j=1}^{n} \in \mathcal{A}$, and $\left(h_{j}\right)_{j=1}^{n}$ $\in \mathcal{H}$, we have

$$
\sum_{i, j=1}^{n}\left\langle\varphi\left(a_{j}^{*} a_{i}\right) h_{i}, h_{j}\right\rangle \geq 0 .
$$

Since for any $\left(a_{j}\right)_{j=1}^{n} \in \mathcal{A}$ the matrix $\left[a_{j}^{*} a_{i}\right]_{i, j=1}^{n}$ is a non-negative element in $M_{n}(\mathcal{A})$, if $\varphi$ is positive definite then it is completely positive. Conversely, because any positive element in $M_{n}(\mathcal{A})$ can be written as a sum of elements of type $\left[a_{j}^{*} a_{i}\right]_{i, j=1}^{n}$, it follows that complete positivity is the same with positive definiteness.

$\mathrm{CP}(\mathcal{A} ; \mathcal{H})$ denotes the set of all completely positive maps from $\mathcal{A}$ into $\mathcal{B}(\mathcal{H})$. If $\varphi, \psi$ $\in \operatorname{CP}(\mathcal{A} ; \mathcal{H})$ one writes $\varphi \leq \psi$ if $\psi-\varphi \in \operatorname{CP}(\mathcal{A} ; \mathcal{H})$; this is the natural partial order (reflexive, antisymmetric, and transitive) on the cone $\mathrm{CP}(\mathcal{A} ; \mathcal{H})$. With respect to the partial order relation $\leq$, $\mathrm{CP}(\mathcal{A} ; \mathcal{H})$ is a strict convex cone.

Given $\theta \in \mathrm{CP}(\mathcal{A} ; \mathcal{H})$ we consider its minimal Stinespring representation $\left(\pi_{\theta} ; \mathcal{K}_{\theta} ; V_{\theta}\right)$ (cf. Stinespring ${ }^{34}$ ). Recall that $\mathcal{K}_{\theta}$ is the Hilbert space quotient completion of the algebraic tensor product of the linear space $\mathcal{A} \otimes \mathcal{H}$ endowed with the inner product,

$$
\langle a \otimes h, b \otimes k\rangle_{\theta}=\left\langle\theta\left(b^{*} a\right) h, k\right\rangle \quad \text { for all } a, b \in \mathcal{A}, \quad h, k \in \mathcal{H} .
$$

$\pi_{\theta}$ is defined on elementary tensors by $\pi_{\theta}(a)(b \otimes h)=(a b) \otimes h$ for all $a, b \in \mathcal{A}$ and $h \in \mathcal{H}$, and then extended by linearity and continuity to a $*$-representation $\pi_{\theta}: \mathcal{A} \rightarrow \mathcal{K}_{\theta}$. Also, $V_{\theta} h=[1 \otimes h]_{\theta} \in \mathcal{K}_{\theta}$ for all $h \in \mathcal{H}$, where $[a \otimes h]_{\theta}$ denotes the equivalence class in the factor space $\mathcal{A} \otimes \mathcal{H} / \mathcal{N}_{\theta}$, and $\mathcal{N}_{\theta}$ is the isotropic subspace corresponding to the inner product $\langle\cdot, \cdot\rangle_{\theta}$. The minimal Stinespring rep- 
resentation $\left(\pi_{\theta} ; \mathcal{K}_{\theta} ; V_{\theta}\right)$ of $\theta$ is uniquely defined, modulo unitary equivalence, subject to the following conditions.

(i) $\quad \mathcal{K}_{\theta}$ is a Hilbert space and $V_{\theta} \in \mathcal{B}\left(\mathcal{H}, \mathcal{K}_{\theta}\right)$.

(ii) $\pi_{\theta}$ is a *-representation of $\mathcal{A}$ on $\mathcal{K}_{\theta}$ such that $\theta(a)=V_{\theta}^{*} \pi_{\theta}(a) V_{\theta}$ for all $a \in \mathcal{A}$.

(iii) $\pi_{\theta}(\mathcal{A}) V_{\theta} \mathcal{H}$ is total in $\mathcal{K}_{\theta}$.

In case $\theta$ is unital, the linear operator $V_{\theta}$ is an isometry and hence, due to the uniqueness, one usually replaces $V$ with the canonical embedding $\mathcal{H} \hookrightarrow \mathcal{K}$. This is explains why the Stinespring representation theorem is considered to be a "dilation-type result," and the minimal Stinespring representation is sometimes called the minimal Stinespring dilation, cf. Ref. 29 and the bibliography cited there.

We record briefly the well-known example of non-negative Borel measures on compact Hausdorff spaces, for later use.

Example 2.1: Let $X$ be a compact Hausdorff space and let $C(X)$ denote the unital Abelian $C^{*}$-algebra of complex valued continuous functions on $X$. If $\mu$ is a finite non-negative Borel measure on $X$ then it can be viewed as a bounded linear map $\mu: C(X) \rightarrow \mathrm{C}$,

$$
\mu(f)=\int_{X} f(x) d \mu(x), \quad f \in C(X) .
$$

Consider the Hilbert space $\mathcal{K}_{\mu}=L^{2}(X ; \mu)$ and the canonical representation $\pi_{\mu}$ of $C(X)$ on $L^{2}(X ; \mu)$ defined by $\pi_{\mu}(f)=M_{f}$, where $M_{f} \in \mathcal{B}\left(L^{2}(X ; \mu)\right)$ is the operator of multiplication with $f$. Let $V_{\mu}: \mathrm{C} \rightarrow L^{2}(X ; \mu)$ be the linear operator that maps each complex number $z$ to the constant function on $X$ with value $z$. Then $\left(\pi_{\mu} ; \mathcal{K}_{\mu} ; V_{\mu}\right)$ is the minimal Stinespring representation of $\mu$, in particular, $\mu$ is completely positive.

\section{B. Radon-Nikodym derivatives}

Let $\varphi, \theta \in \operatorname{CP}(\mathcal{A} ; \mathcal{H})$ be such that $\varphi \leq \theta$ and consider the minimal Stinespring representation $\left(\pi_{\varphi} ; \mathcal{K}_{\varphi} ; V_{\varphi}\right)$ of $\varphi$, and similarly for $\theta$. Then the identity operator $J_{\varphi, \theta}: \mathcal{A} \otimes \mathcal{H} \rightarrow \mathcal{A} \otimes \mathcal{H}$ has the property that $J_{\varphi, \theta} \mathcal{N}_{\theta} \subseteq \mathcal{N}_{\varphi}$, hence it can be factored to a linear operator $J_{\varphi, \theta}:(\mathcal{A} \otimes \mathcal{H}) / \mathcal{N}_{\theta} \rightarrow(\mathcal{A}$ $\otimes \mathcal{H}) / \mathcal{N}_{\varphi}$ and then can be extended by continuity to a contractive linear operator $J_{\varphi, \theta}$ $\in \mathcal{B}\left(\mathcal{K}_{\theta}, \mathcal{K}_{\varphi}\right)$. It is easy to see that

$$
J_{\theta, \varphi} V_{\theta}=V_{\varphi},
$$

and that

$$
J_{\theta, \varphi} \pi_{\theta}(a)=\pi_{\varphi}(a) J_{\theta, \varphi} \text { for all } a \in \mathcal{A}
$$

Thus, letting

$$
D_{\theta}(\varphi):=J_{\theta, \varphi}^{*} J_{\theta, \varphi}
$$

we get a contractive linear operator in $\mathcal{B}\left(\mathcal{K}_{\theta}\right)$. In addition, as a consequence of (2.6), $D_{\theta}(\varphi)$ commutes with all operators $\pi_{\theta}(a)$ for $a \in \mathcal{A}$, briefly, $D_{\theta}(\varphi) \in \pi_{\theta}(\mathcal{A})^{\prime}$ [given a subset $\mathcal{T}$ of $B(\mathcal{H})$ we write $\mathcal{T}^{\prime}=\{B \in \mathcal{B}(\mathcal{H}) \mid A B=B A$ for all $A \in \mathcal{T}\}$ for the commutant of $\left.\mathcal{T}\right]$ and

$$
\varphi(a)=V_{\theta}^{*} D_{\theta}(\varphi) \pi_{\theta}(a) V_{\theta}=V_{\theta}^{*} D_{\theta}(\varphi)^{1 / 2} \pi_{\theta}(a) D_{\theta}(\varphi)^{1 / 2} V_{\theta} \text { for all } a \in \mathcal{A} .
$$

The property (2.8) uniquely characterizes the operator $D_{\theta}(\varphi)$. The operator $D_{\theta}(\varphi)$ is called the Radon-Nikodym derivative of $\varphi$ with respect to $\theta$.

It is immediate from (2.8) that, for any $n \in \mathbb{N},\left(a_{j}\right)_{j=1}^{n} \in \mathcal{A}$, and $\left(h_{j}\right)_{j=1}^{n} \in \mathcal{H}$, the following formula holds: 


$$
\sum_{i, j=1}^{n}\left\langle\varphi\left(a_{j}^{*} a_{i}\right) h_{i}, h_{j}\right\rangle=\left\|D_{\theta}(\varphi)^{1 / 2} \sum_{j=1}^{n} \pi_{\theta}\left(a_{j}\right) V_{\theta} h_{j}\right\|^{2} .
$$

This shows that for any $\varphi, \psi \in \operatorname{CP}(\mathcal{A} ; \mathcal{H})$ with $\varphi, \psi \leq \theta$, we have $\varphi \leq \psi$ if and only if $D_{\theta}(\varphi)$ $\leq D_{\theta}(\psi)$.

In addition, if $\varphi, \psi \in \operatorname{CP}(\mathcal{A} ; \mathcal{H})$ are such that $\varphi, \psi \leq \theta$ then for any $t \in[0,1]$ the completely positive map $(1-t) \varphi+t \psi$ is $\leq \theta$ and

$$
D_{\theta}((1-t) \varphi+t \psi)=(1-t) D_{\theta}(\varphi)+t D_{\theta}(\psi) .
$$

The above considerations can be summarized in the following.

Theorem 2.2: [Arveson (Ref. 6)] Let $\theta \in \operatorname{CP}(\mathcal{A} ; \mathcal{H})$. The mapping $\varphi \mapsto D_{\theta}(\varphi)$ defined in (2.7), with its inverse given by (2.8), is an affine and order-preserving isomorphism between the convex and partially ordered sets $(\{\varphi \in \mathrm{CP}(\mathcal{A} ; \mathcal{H}) \mid \varphi \leq \theta\} ; \leq)$ and $\left(\left\{A \in \pi_{\theta}(\mathcal{A})^{\prime} \mid 0 \leq A \leq I\right\} ; \leq\right)$.

One says that $\psi$ uniformly dominates $\varphi$, and we write $\varphi \leq_{\mathrm{u}} \psi$, if for some $t>0$ we have $\varphi$ $\leq t \psi$. This is a partial preorder relation (only reflexive and transitive). The associated equivalence relation (we can call it uniform equivalence) is denoted by $\simeq_{u}$, that is, for $\varphi, \psi \in \operatorname{CP}(\mathcal{A} ; \mathcal{H})$ we have $\varphi \simeq_{u} \psi$ if and only if $\varphi \leq_{u} \psi \leq_{u} \varphi$. It is immediate from Theorem 2.2 the following.

Corollary 2.3: For a given $\theta \in \operatorname{CP}(\mathcal{A} ; \mathcal{H})$, the mapping $\varphi \mapsto D_{\theta}(\varphi)$ defined in (2.7), with its inverse given by (2.8), is an affine and order-preserving isomorphism between the convex cones $\left(\left\{\varphi \in \mathrm{CP}(\mathcal{A} ; \mathcal{H}) \mid \varphi \leq_{\mathrm{u}} \theta\right\} ; \leq\right)$ and $\left(\left\{A \in \pi_{\theta}(\mathcal{A})^{\prime} \mid 0 \leq A\right\} ; \leq\right)$.

We also record the example of the classical Radon-Nikodym derivative, from the perspective of Arveson's Radon-Nikodym derivative.

Example 2.4: Let $\mu$ be a finite non-negative Borel measure on the compact Hausdorff space $X$ and consider its minimal Stinespring representation $\left(\pi_{\mu} ; \mathcal{K}_{\mu} ; V_{\mu}\right)$ as in Example 2.1. Let $\nu$ be another finite non-negative Borel measure on $X$ such that, when viewing these measures as bounded linear functional on $C(X)$ as in (2.4), we have $\nu \leq_{\mathrm{u}} \mu$, that is, for some $t>0$ we have

$$
\int_{X} h(x) d \nu(x) \leq t \int_{X} h(x) d \mu(x), \quad h \in C(X),
$$

equivalently, $\nu(E) \leq t \mu(E)$ for all $E$ Borel subsets of $X$. Note that the commutant $\pi_{\mu}(C(X))^{\prime}$ coincides with the maximal Abelian von Neumann algebra $\mathcal{M}=\left\{M_{g} \mid g \in L^{\infty}(X ; \mu)\right\}$, where $M_{g}$ is the bounded operator of multiplication on $L^{2}(X ; \mu)$ with the function $g$ in the $C^{*}$-algebra (actually, a $W^{*}$-algebra) $L^{\infty}(X ; \mu)$ of $\mu$-essentially bounded functions on $X$.

By Corollary 2.3, $D_{\mu}(\nu)$, the Radon-Nikodym derivative of $\nu \in \mathrm{CP}(C(X) ; \mathrm{C})$ with respect to $\mu \in \mathrm{CP}(C(X) ; \mathrm{C})$, is a non-negative operator in the von Neumann algebra $\mathcal{M}$ and hence, there exists uniquely $f \in L^{\infty}(X ; \mu)$ such that $D_{\mu}(\nu)=M_{f}$.

On the other hand, the classical Radon-Nikodym derivative $d \nu / d \mu$ is originally a $\mu$-absolutely integrable function on $X$ such that $d \nu=f d \mu$, but it is actually $\mu$-essentially bounded, with the $\mu$-essential supremum $\|f\|_{\infty} \leq t$, because of (2.11). It is easy to see that $f=d \nu / d \mu \mu$-a.e., due to the uniqueness of both Radon-Nikodym derivatives.

\section{Absolute continuity}

Given $\varphi, \psi \in \operatorname{CP}(\mathcal{A} ; \mathcal{H})$, we say that $\psi$ is $\varphi$-absolutely continuous, and we write $\psi \ll \varphi$, if there exists a sequence $\psi_{n} \in \mathrm{CP}(\mathcal{A} ; \mathcal{H})$ subject to the following conditions.

(acp1) The sequence $\left(\psi_{n}\right)$ is nondecreasing, that is, $\psi_{n} \leq \psi_{n+1}$ for all $n \in \mathbb{N}$.

(acp2) $\mathrm{SO}-\lim \psi_{n}(a)=\psi(a)$ for all $a \in \mathcal{A}$.

(acp3) $\psi_{n} \leq_{\mathrm{u}} \varphi$ for all $n \in \mathbb{N}$, more precisely, for each $n \in \mathbb{N}$ there exists $t_{n}>0$ such that $\psi_{n}$ $\leq t_{n} \varphi$.

This definition is inspired from the definition of absolute continuity for positive definite functions on *-semigroups given by Ando and Szymański in Ref. 5.

The following lemma is the analog of Proposition 2.5 in Ref. 5. 
Lemma 2.5: For a given $\theta \in \mathrm{CP}(\mathcal{A} ; \mathcal{H})$, let $\psi_{n}, \psi \in \mathrm{CP}(\mathcal{A} ; \mathcal{H}), \psi_{n} \leq \psi \leq_{u} \theta$, and $\psi_{n} \leq \psi_{n+1}$ for all $n \in \mathbb{N}$. Then the following are equivalent.

(i) $\mathrm{SO}-\lim _{n \rightarrow \infty} D_{\theta}\left(\psi_{n}\right)=D_{\theta}(\psi)$.

(ii) For all $a \in \mathcal{A}$ SO- $\lim _{n \rightarrow \infty} \psi_{n}(a)=\psi(a)$, that is,

$$
\lim _{n \rightarrow \infty}\left\|\psi_{n}(a) h-\psi(a) h\right\| \quad \text { for all } a \in \mathcal{A}, \quad h \in \mathcal{H} .
$$

(iii) For all $a \in \mathcal{A}$, WO- $\lim _{n \rightarrow \infty} \psi_{n}(a)=\psi(a)$, that is,

$$
\lim _{n \rightarrow \infty}\left\langle\psi_{n}(a) h, k\right\rangle=\langle\psi(a) h, k\rangle \quad \text { for all } a \in \mathcal{A}, \quad h, k \in \mathcal{H} .
$$

Proof: If $D_{\theta}\left(\psi_{n}\right)$ converges SO to $D_{\theta}(\psi)$ then, by (2.8), for all $a \in \mathcal{A}$ and $h \in \mathcal{H}$, we have

$$
\lim _{n \rightarrow \infty}\left\|\psi_{n}(a) h-\psi(a) h\right\|=\lim _{n \rightarrow \infty}\left\|V_{\theta}^{*}\left(D_{\theta}\left(\psi_{n}\right)-D_{\theta}(\psi)\right) \pi(a) V_{\theta} h\right\|=0,
$$

hence (ii) holds.

Since, clearly, (ii) implies (iii), it remains to prove that (iii) implies (i). To see this, note first that, by considering the sequence of completely positive maps $\psi-\psi_{n}$, it is enough to prove the implication in case $\psi=0$. Then, by (2.9) we get that the sequence $\left(D_{\theta}\left(\psi_{n}\right)^{1 / 2}\right)$ converges strongly on the subspace generated by $\pi(\mathcal{A}) V_{\theta} \mathcal{H}$, which by minimality is dense in $\mathcal{K}_{\theta}$ and, taking into account that all the operators $D_{\theta}\left(\psi_{n}\right)$ are contractions, it follows by a standard argument in operator theory that $\left(D_{\theta}\left(\psi_{n}\right)\right)$ converges strongly to 0 .

In the following we use the definition of absolute continuity of non-negative operators as in the paragraph below Theorem 6.2 in Appendix A 2. From Corollary 2.3 and Lemma 2.5 one gets the following analog of Proposition 2.7 in Ref. 5.

Proposition 2.6: For a given $\theta \in \mathrm{CP}(\mathcal{A} ; \mathcal{H})$, let $\varphi, \psi \in \mathrm{CP}(\mathcal{A} ; \mathcal{H})$ be such that $\varphi, \psi \leq_{\mathrm{u}} \theta$. Then $\psi$ is $\varphi$-absolutely continuous if and only if $D_{\theta}(\psi)$ is $D_{\theta}(\varphi)$-absolutely continuous.

We also record the following basic properties of the relation of absolute continuity.

Proposition 2.7: Let $\varphi \in \mathrm{CP}(\mathcal{A} ; \mathcal{H})$.

(i) If $\psi, \rho \in \mathrm{CP}(\mathcal{A} ; \mathcal{H})$ are $\varphi$-absolutely continuous, then the same is $t \psi+s \rho$ for all $t, s \geq 0$.

(ii) Assume that $\left(\psi_{n}\right)$ is a sequence in $\mathrm{CP}(\mathcal{A} ; \mathcal{H})$ subject to the following properties.

(1) The sequence $\left(\psi_{n}\right)$ is nondecreasing.

(2) There exists $\psi \in \mathrm{CP}(\mathcal{A} ; \mathcal{H})$ such that $\psi(a)=\operatorname{SO} \lim _{n \rightarrow \infty} \psi_{n}(a)$ for all $a \in \mathcal{A}$.

(3) For all $n \in \mathbb{N}, \psi_{n}$ is $\varphi$-absolutely continuous.

Then $\psi$ is $\varphi$-absolutely continuous.

(iii) If $\psi \simeq{ }_{\mathrm{u}} \rho$ then $\psi$ is $\varphi$-absolutely continuous if and only if $\rho$ is $\varphi$-absolutely continuous.

The notion of absolute continuity for operator valued completely positive maps is a generalization of the notion of absolute continuity for positive measures. Among the different ways of proving this fact, one may choose the way passing through Example 2.4 and Proposition 2.6.

Proposition 2.8: Let $X$ be a Hausdorff compact space and $\mu, \nu$ be two non-negative finite Borel measures on $X$, considered as completely positive maps $\mu, \nu: C(X) \rightarrow \mathrm{C}$ as in (2.4). Then the following assertions are equivalent.

(1) $\nu \ll \mu$.

(2) For every Borel subset $A \subseteq X$ such that $\mu(A)=0$ it follows $\nu(A)=0$ (that is, the classical notion of absolute continuity). 


\section{Recovering the minimal Stinespring representation}

Let $\varphi, \theta \in \operatorname{CP}(\mathcal{A} ; \mathcal{H})$ be such that $\varphi \leq_{\mathrm{u}} \theta$ and, as in Sec. II C, let $\left(\pi_{\theta} ; \mathcal{K}_{\theta} ; V_{\theta}\right)$ be the minimal Stinespring representation for $\theta$. Let $D_{\theta}(\varphi)$ be the Radon-Nikodym derivative of $\varphi$ with respect to $\theta$ as in (2.7). Then, with respect to the decomposition $\mathcal{K}_{\theta}=\operatorname{Ker}\left(D_{\theta}(\varphi)\right) \oplus\left(\mathcal{K}_{\theta} \ominus \operatorname{Ker}\left(D_{\theta}(\varphi) x\right)\right)$,

$$
V_{\theta}=\left[\begin{array}{l}
V_{\theta, 1} \\
V_{\theta, 2}
\end{array}\right], \quad \pi_{\theta}(a)=\left[\begin{array}{cc}
\pi_{\theta, 1}(a) & 0 \\
0 & \pi_{\theta, 2}(a)
\end{array}\right] \quad \text { for all } a \in \mathcal{A},
$$

where the diagonal form of $\pi(a)$ follows because it commutes with $D_{\theta}(\varphi)$. Clearly,

$$
\varphi(a)=\left(D_{\theta}(\varphi)^{1 / 2} P_{\mathcal{K}_{\theta} \ominus \operatorname{Ker}\left(D_{\theta}(\varphi)\right)} V_{\theta, 2}\right)^{*} \pi_{\theta, 2}(a) P_{\mathcal{K}_{\theta} \ominus \operatorname{Ker}\left(D_{\theta}(\varphi)\right)} D_{\theta}(\varphi)^{1 / 2} V_{\theta, 2}, \quad a \in \mathcal{A},
$$

and from here it follows that the triple $\left(\pi_{\theta, 2} ; \mathcal{K}_{\theta} \ominus \operatorname{Ker}\left(D_{\theta}(\varphi)\right) ; P_{\mathcal{H} \ominus \operatorname{Ker}\left(D_{\theta}(\varphi)\right)} D_{\theta}(\varphi)^{1 / 2} V_{\theta, 2}\right)$ is a Stinespring representation for $\varphi$. The minimality follows from the observation that the set $\pi_{\theta, 2}(\mathcal{A}) V_{\theta, 2} \mathcal{H}$ is total in $\mathcal{H} \ominus \operatorname{Ker}\left(D_{\theta}(\varphi)\right)$. Thus, we have the following.

Theorem 2.9: Under the previous assumptions, the triple

$$
\left(P_{\mathcal{K}_{\theta} \ominus \operatorname{Ker}\left(D_{\theta}(\varphi)\right)} \pi_{\theta} P_{\mathcal{K}_{\theta} \ominus \operatorname{Ker}\left(D_{\theta}(\varphi)\right)} ; \mathcal{K}_{\theta} \ominus \operatorname{Ker}\left(D_{\theta}(\varphi)\right) ; P_{\mathcal{K}_{\theta} \ominus \operatorname{Ker}\left(D_{\theta}(\varphi)\right)} D_{\theta}(\varphi)^{1 / 2} P_{\mathcal{K}_{\theta} \ominus \operatorname{Ker}\left(D_{\theta}(\varphi)\right)} V_{\theta}\right)
$$

is the minimal Stinespring representation for $\varphi$.

When $\varphi$ and $\psi$ are also unital then the above theorem coincides with Theorem 4.1 in Ref. 28.

\section{E. Chain rule}

Let $\varphi, \psi, \theta$ be $\mathcal{B}(\mathcal{H})$-valued completely positive maps on the $C^{*}$-algebra $\mathcal{A}$ such that $\varphi \leq_{\mathrm{u}} \psi$ $\leq_{\mathrm{u}} \theta$. Let us consider the minimal Stinespring representations $\left(\pi_{\theta} ; \mathcal{K}_{\theta} ; V_{\theta}\right),\left(\pi_{\psi} ; \mathcal{K}_{\psi} ; V_{\psi}\right)$, and $\left(\pi_{\varphi} ; \mathcal{K}_{\varphi} ; V_{\varphi}\right)$, of $\theta, \psi$, and, respectively, $\varphi$. By the definition of the bounded linear operators $J_{\varphi, \psi}$, $J_{\psi, \theta}$, and $J_{\varphi, \theta}$ (see Sec. II B) it follows that

$$
J_{\varphi, \theta}=J_{\varphi, \psi} J_{\psi, \theta},
$$

hence

$$
D_{\theta}(\varphi)=J_{\varphi, \theta}^{*} J_{\varphi, \theta}=J_{\psi, \theta}^{*} J_{\varphi, \psi}^{*} J_{\varphi, \psi} J_{\psi, \theta}=J_{\psi, \theta}^{*} D_{\psi}(\varphi) J_{\psi, \theta} .
$$

The formula (2.12) is called the chain rule in. Ref. 8.

We now consider all the Radon-Nikodym derivatives $D_{\psi}(\varphi), D_{\theta}(\psi)$, and $D_{\theta}(\varphi)$ and ask for the relation between them, an analog of the chain rule for the Radon-Nikodym derivatives of functions. In order to simplify the notation, let $P=P_{\mathcal{K}_{\theta} \ominus \operatorname{Ker}\left(D_{\theta}(\psi)\right)}$. By Theorem 2.9, we have the following identifications:

$$
\mathcal{K}_{\psi}=\mathcal{K}_{\theta} \ominus \operatorname{Ker}\left(D_{\theta}(\psi)\right), \quad \pi_{\psi}=P \pi_{\theta} P, \quad V_{\psi}=P D_{\theta}(\psi)^{1 / 2} V_{\theta} .
$$

By (2.8), for any $a \in \mathcal{A}$ we have

$$
\begin{aligned}
\varphi(a) & =V_{\psi} D_{\psi}(\varphi)^{1 / 2} \pi_{\psi}(a) D_{\psi}(\varphi)^{1 / 2} V_{\psi}=V_{\theta}^{*} P D_{\theta}(\psi)^{1 / 2} P D_{\psi}(\varphi)^{1 / 2} P \pi_{\theta}(a) P D_{\theta}(\psi)^{1 / 2} P V_{\theta} \\
& =V_{\theta}^{*} P D_{\theta}(\psi)^{1 / 2} P D_{\psi}(\varphi) D_{\theta}(\psi)^{1 / 2} P \pi_{\theta}(a) P V_{\theta} .
\end{aligned}
$$

From these calculations and taking into account the uniqueness of the Radon-Nikodym derivative it follows:

Theorem 2.10: Let $\varphi, \psi, \theta \in \mathrm{CP}(\mathcal{A} ; \mathcal{H})$ be such that $\varphi \leq_{\mathrm{u}} \psi \leq_{\mathrm{u}} \theta$. Then, modulo the identification of $\mathcal{K}_{\psi}$ as in (2.13), we have

$$
D_{\theta}(\varphi)=D_{\theta}(\psi)^{1 / 2} P_{\mathcal{K}_{\theta} \ominus \operatorname{Ker}\left(D_{\theta}(\psi)\right)} D_{\psi}(\varphi) P_{\mathcal{K}_{\theta} \ominus \operatorname{Ker}\left(D_{\theta}(\psi)\right)} D_{\theta}(\psi)^{1 / 2} .
$$

Actually, the chain rule in Theorem 2.10 can be obtained directly from (2.12), as follows. By the definition of the Radon-Nikodym derivative as in (2.7), it follows that $J_{\psi, \theta}=W D_{\theta}(\psi)^{1 / 2}$, where 
$W \in \mathcal{B}\left(\mathcal{K}_{\theta}, \mathcal{K}_{\psi}\right)$ is a partial isometry with $\operatorname{Ker}(W)=\operatorname{Ker}\left(D_{\theta}(\psi)\right)$ and surjective (because $J_{\psi, \theta}$ has dense range). But, the meaning of the identification as in (2.12) is that there exists a unitary operator $U \in \mathcal{B}\left(\mathcal{K}_{\psi}, \mathcal{K}_{\theta} \ominus \operatorname{Ker}\left(D_{\theta}(\psi)\right)\right)$ such that $W^{*}=P_{\mathcal{K}_{\theta} \ominus \operatorname{Ker}\left(D_{\theta}(\psi)\right)} U$. Thus, from (2.12), modulo the identification as in (2.12), we get (2.13).

\section{F. Radon-Nikodym derivatives for absolutely continuous completely positive maps}

The first task of this subsection is to consider the extension of Radon-Nikodym derivatives to relatively absolutely continuous completely positive maps that will be, in general, unbounded operators. This can be defined in two ways, as shown in Ref. 9 and, respectively, Ref. 28, and we show that, actually, these two methods produce the same definition.

We derive the main result from a comparison theory for quadratic forms, whose details are given in the Appendix B. In our opinion, we consider this as the natural framework to deal with this kind of questions. One of the advantages is that, in this way, a spectral approximation of the Radon-Nikodym derivative is also obtained: this is the second task of this subsection.

Thus, recalling that our setting is that of $\mathcal{B}(\mathcal{H})$-valued completely positive maps on a $C^{*}$-algebra $\mathcal{A}$, consider the vector space $\mathcal{V}=\mathcal{A} \otimes \mathcal{H}$, the algebraic tensor product. To any $\varphi$ $\in \operatorname{CP}(\mathcal{A} ; \mathcal{H})$ we associate the quadratic form $q_{\varphi}$ with

$$
q_{\varphi}\left(\sum_{j=1}^{n} a_{j} \otimes h_{j}\right)=\sum_{i, j=1}^{n}\left\langle\varphi\left(a_{j}^{*} a_{i}\right) h_{i}, h_{j}\right\rangle,
$$

which is non-negative by (2.2). Thus, the formula (2.14) establishes an affine and order-preserving embedding of $\mathrm{CP}(\mathcal{A} ; \mathcal{H})$ into $\mathbf{Q}(\mathcal{V})^{+}$(see Appendix B 1 for notation).

To this end, we recall the following definition: given a von Neumann algebra $\mathcal{M} \subseteq \mathcal{B}(\mathcal{H})$, a densely defined linear operator $T$ in $\mathcal{H}$ is affiliated to $\mathcal{M}$, and we write $T \eta \mathcal{M}$, if for any operator $X \in \mathcal{M}^{\prime}$ we have $X T \subseteq T X$, more precisely, $\operatorname{Dom}(T)$ is invariant under $X$ and $X T h=T X h$ for all $h \in \operatorname{Dom}(T)$. If $T$ is a (unbounded) self-adjoint operator in $\mathcal{H}$, then $T \eta \mathcal{M}$ if and only all the spectral projections of $T$ are in $\mathcal{M}$.

Also, given a closable operator $T$ in the Hilbert space, a subspace $\mathcal{D} \subseteq \operatorname{Dom}(T)$ is called a core for $T$ if the closure of $T \mid \mathcal{D}$ coincides with the closure of $T$.

Theorem 2.11: Let $\varphi, \psi \in \mathrm{CP}(\mathcal{A} ; \mathcal{H})$ and let $\left(\pi_{\varphi} ; \mathcal{K}_{\varphi} ; V_{\varphi}\right)$ be the minimal Stinespring representation of $\varphi$. Then, $\psi$ is $\varphi$-absolutely continuous if and only if there exists a (generally unbounded) linear operator $D_{\varphi}(\psi)$, uniquely determined by the following properties.

(i) $D_{\varphi}(\psi)$ is a positive selfadjoint operator in $\mathcal{K}_{\varphi}$ and it is affiliated with $\pi_{\varphi}(\mathcal{A})^{\prime}$.

(ii) $\operatorname{Lin}\left\{\pi_{\varphi}(\mathcal{A}) V_{\varphi} \mathcal{H}\right\}$ is a core for $D_{\varphi}(\psi)^{1 / 2}$ and $D_{\varphi}(\psi)^{1 / 2} V_{\varphi}$ is bounded.

(iii) $\quad \psi(a)=\left(D_{p}(q)^{1 / 2} V_{\varphi}\right)^{*} \pi(a) D_{p}(q)^{1 / 2} V_{\varphi}$ for all $a \in \mathcal{A}$.

Proof: The technicalities of the proof have been deferred to Appendix B. Briefly, if for arbitrary $\varphi \in \mathrm{CP}(\mathcal{A} ; \mathcal{H})$ we consider its Stinespring representation $\left(\pi_{\varphi} ; \mathcal{K}_{\varphi} ; V_{\varphi}\right)$ then $\left(\mathcal{K}_{\varphi} ; \Pi_{\varphi}\right)$ is a Hilbert space induced by $q_{\varphi}$, with the definition in Appendix B 1, where

$$
\Pi_{\varphi}\left(\sum_{j=1}^{n} a_{j} \otimes h_{j}\right)=\sum_{j=1}^{n} \pi\left(a_{j}\right) h_{j} .
$$

These observations show that we can first consider the underlying comparison theory for nonnegative quadratic forms as in Appendix B with the important difference that, for the case of completely positive maps, everything concerning the Radon-Nikodym derivatives should correspond to operators in the von Neumann algebra $\pi_{\varphi}(\mathcal{A})^{\prime}$. Having this in mind, we thus can apply Theorem 7.13(a) in Appendix A.

Theorem 2.11 is essentially Corollary 4.2 in Ref. 28. Theorem 7.7 shows that the cited corollary is equivalent with the main result in Ref. 9. 
Since the Radon-Nikodym derivative of absolutely continuous positive definite maps is, in general, an unbounded operator, there is a natural need for its approximation with bounded Radon-Nikodym derivatives. Let $\left(A_{n}\right)$ be a sequence of (generally, unbounded) positive selfadjoint operators in a Hilbert space $\mathcal{H}$. One says that $\left(A_{n}\right)$ converges in the strong resolvent sense to the positive self-adjoint operator $A$ in $\mathcal{H}$ if SO- $\lim _{n \rightarrow \infty}\left(I+A_{n}\right)^{-1}=(I+A)^{-1}$. The name is justified by the known fact that, in this case, we also have SO- $\lim _{n \rightarrow \infty}\left(\zeta I-A_{n}\right)^{-1}=(\zeta I-A)^{-1}$ for all $\zeta$ $\in \mathrm{C} \backslash \mathrm{R}$ (e.g., see Corollary VIII.1.4 in Ref. 20). The same approach as in the proof of Theorem 2.11 can be used in order to apply Theorem 7.13(b) and get the following.

Theorem 2.12: Let $\varphi, \psi \in \operatorname{CP}(\mathcal{A} ; \mathcal{H})$ and let $\left(\pi_{\varphi} ; \mathcal{K}_{\varphi} ; V_{\varphi}\right)$ be the minimal Stinespring representation of $\varphi$.

Assume that $\psi$ is $\varphi$-absolutely continuous. Then, for any sequence $\left(\psi_{n}\right)$ of maps in $\operatorname{CP}(\mathcal{A} ; \mathcal{H})$ that is nondecreasing, $\mathrm{SO}-\lim _{n \rightarrow \infty} \psi_{n}(a)=\psi(a)$ for all $a \in \mathcal{A}$, and $\psi_{n} \leq_{\mathrm{u}} \varphi$ for all $n \in \mathbb{N}$, it follows that $D_{\varphi}\left(\psi_{n}\right)$ converges to $D_{\varphi}(\psi)$ in the strong resolvent sense.

\section{LEBESGUE DECOMPOSITIONS FOR COMPLETELY POSITIVE MAPS}

\section{A. The maximal Lebesgue decomposition}

Let $\varphi$ and $\psi$ be two completely positive maps from $\mathcal{A}$ into $\mathcal{B}(H) . \varphi$ is called $\psi$-singular if the only map $\rho \in \operatorname{CP}(\mathcal{A} ; \mathcal{H})$ such that $\rho \leq \varphi, \psi$ is 0 . Note that $\varphi$ is $\psi$-singular if and only if $\psi$ is $\varphi$-singular and, in this case, we call $\varphi$ and $\psi$ mutually singular.

A decomposition $\varphi=\varphi_{1}+\varphi_{0}$, where $\varphi_{1}, \varphi_{0} \in \mathrm{CP}(\mathcal{A} ; \mathcal{H}), \varphi_{1}$ is $\psi$-absolutely continuous and $\varphi_{0}$ is $\psi$-singular, is called a $\psi$-Lebesgue decomposition of $\varphi$.

Theorem 3.1: Let $\varphi, \psi \in \mathrm{CP}(\mathcal{A} ; \mathcal{H})$. There exists a $\psi$-Lebesgue decomposition of $\varphi=\varphi_{\mathrm{ac}}+\varphi_{\mathrm{s}}$ such that $\varphi_{\mathrm{ac}}$ is maximal among all $\psi$-absolutely continuous maps $\rho \in \operatorname{CP}(\mathcal{A} ; \mathcal{H})$ with $\rho \leq \varphi$.

We will get the proof of Theorem 3.1 after proving the following lemma.

Lemma 3.2: Let $F \in \mathcal{B}(K)$ be such that $0 \leq F \leq I$. Then, with the notation as in $(\mathrm{A} 4)$,

$$
[F](I-F)=P_{\mathcal{H} \ominus \operatorname{Ker}(F)}(I-F) .
$$

Proof: It is easy to see that

$$
\operatorname{Ran}(F) \subseteq\left\{h \in \mathcal{K} \mid(I-F)^{1 / 2} h \in \operatorname{Ran}\left(F^{1 / 2}\right)\right\} \subseteq \mathcal{H} \ominus \operatorname{Ker}(F),
$$

hence, by Theorem 6.1 it follows that $P_{F, I-F}=P_{\mathcal{K} \ominus \operatorname{Ker}(F)}$ and then

$$
[F](I-F)=(I-F)^{1 / 2} P_{\mathcal{K} \ominus \operatorname{Ker}(F)}(I-F)^{1 / 2}=(I-F) P_{\mathcal{K} \ominus \operatorname{Ker}(F)} .
$$

Proof of Theorem 3.1: To simplify the notation, let $(\pi, \mathcal{K}, V)$ be the minimal Stinespring representation for $\theta:=\varphi+\psi$. Then the Radon-Nikodym derivatives $D_{\theta}(\psi)$ and $D_{\theta}(\varphi)$ in $\mathcal{B}(\mathcal{K})$ satisfy the following relation:

$$
D_{\theta}(\varphi)+D_{\theta}(\psi)=I \text {. }
$$

Define the linear mappings $\varphi_{\mathrm{ac}}, \varphi_{\mathrm{s}}: \mathcal{A} \rightarrow \mathcal{B}(\mathcal{H})$ by

$$
\varphi_{\mathrm{ac}}(a)=V^{*} D_{\theta}(\varphi) P_{\mathcal{K} \ominus \operatorname{Ker}\left(D_{\theta}(\psi)\right)} \pi(a) V \quad \text { and } \quad \varphi_{\mathrm{s}}(a)=V^{*} P_{\operatorname{Ker}\left(D_{\theta}(\psi)\right)} \pi(a) V .
$$

Note that for any $a \in \mathcal{A}$ the operators $\pi(a), D_{\theta}(\varphi)$, and $P_{\mathcal{K} \ominus \operatorname{Ker}\left(D_{\theta}(\psi)\right)}$, mutually commute. It is straightforward to check that $\varphi_{\mathrm{ac}}$ and $\varphi_{\mathrm{s}}$ are completely positive and that $\varphi=\varphi_{\mathrm{ac}}+\varphi_{\mathrm{s}}$.

Clearly, $\varphi_{\mathrm{ac}}, \varphi_{\mathrm{s}} \leq \theta$. We claim that

$$
D_{\theta}\left(\varphi_{\mathrm{ac}}\right)=\left[D_{\theta}(\psi)\right] D_{\theta}(\varphi) .
$$

To see this, we use (3.1) and Lemma 3.2 in order to get 


$$
\left[D_{\theta}(\psi)\right] D_{\theta}(\varphi)=D_{\theta}(\varphi) P_{\mathcal{K} \ominus \operatorname{Ker}\left(D_{\theta}(\psi)\right)} .
$$

Then (3.3) follows by the uniqueness of the Radon-Nikodym derivative, as explained in Sec. II B.

Now, in view of Theorem 2.2, Proposition 2.6, and the definition of $\varphi_{\mathrm{ac}}$ as in (3.2), in order to prove that $\varphi_{\mathrm{ac}}$ is $\psi$-absolutely continuous we have to prove that $D_{\theta}\left(\varphi_{\mathrm{ac}}\right)$ is $D_{\theta}(\psi)$-absolutely continuous. In view of (3.3), the latter is a consequence of Theorem 6.4 part (i).

In addition, again by Theorem 2.2 and Proposition 2.6, in order to prove that $\varphi_{\text {ac }}$ is maximal among all $\psi$-absolutely continuous $\rho \in \operatorname{CP}(\mathcal{A} ; \mathcal{H})$ such that $\rho \leq \varphi$, we have to prove that $D_{\theta}\left(\varphi_{\text {ac }}\right)$ is maximal among all $D_{\theta}(\psi)$-absolutely continuous operators $C \in \mathcal{B}(\mathcal{K})$ such that $C \leq D_{\theta}(\varphi)$. In view of (3.3), the latter is a consequence of Theorem 6.4 part (ii).

Similarly we have

$$
D_{\theta}\left(\varphi_{s}\right)=D_{\theta}(\varphi)-\left[D_{\theta}(\psi)\right] D_{\theta}(\varphi)=P_{\operatorname{Ker}\left(D_{\theta}(\psi)\right)} .
$$

Then, an argument as before, using Theorem 2.2, Proposition 2.6, (3.4), and finally Theorem 6.4, proves that $\varphi_{s}$ is $\psi$-singular.

The $\psi$-Lebesgue decomposition $\varphi=\varphi_{\mathrm{ac}}+\varphi_{s}$ constructed during the proof of Theorem 3.1 is called the maximal $\psi$-Lebesgue decomposition of $\varphi$ and, clearly, it is unique with this property.

A natural question with respect to this maximal Lebesgue decomposition is: to which extent does it depend on the choice of $\theta=\varphi+\psi$ ? Thinking in terms of quantum measurements, the choice of $\theta$ and the minimal Stinespring representation, the dependency should not occur. Indeed, we can get the analog of the formulas (3.3) and (3.4), when $\theta$ is replaced by an arbitrary completely positive map such that both $\varphi$ and $\psi$ are uniformly dominated by $\theta$, and then we can show that the construction of the maximal Lebesgue decomposition does not depend on the choice of $\theta$.

Theorem 3.3: Let $\varphi, \psi$ and $\rho$ be in $\operatorname{CP}(\mathcal{A} ; \mathcal{H})$ such that $\rho$ uniformly dominates $\varphi$ and $\psi$. Let $\varphi=\varphi_{\mathrm{ac}}+\varphi_{\mathrm{s}}$ be the $\psi$-Lebesgue decomposition of $\varphi$ defined at (3.2). Then, the Radon-Nikodym derivatives of $\varphi_{\mathrm{ac}}$ and $\varphi_{s}$, with respect to $\rho$, can be calculated as follows:

$$
D_{\rho}\left(\varphi_{\mathrm{ac}}\right)=\left[D_{\rho}(\psi)\right] D_{\rho}(\varphi) \text { and } D_{\rho}\left(\varphi_{s}\right)=D_{\rho}(\varphi)-\left[D_{\rho}(\psi)\right] D_{\rho}(\varphi) .
$$

In particular, letting $\left(\pi_{\rho} ; \mathcal{K}_{\rho} ; V_{\rho}\right)$ be the minimal Stinespring representation for $\rho$, we have

$$
\varphi_{\mathrm{ac}}(a)=V_{\rho}^{*}\left(\left[D_{\rho}(\psi)\right] D_{\rho}(\varphi)\right) \pi_{\rho}(a) V_{\rho}, \quad a \in \mathcal{A},
$$

and

$$
\varphi_{s}(a)=V_{\rho}^{*}\left(D_{\rho}(\psi)-\left[D_{\rho}(\varphi)\right] D_{\rho}(\varphi)\right) \pi_{\rho}(a) V_{\rho}, \quad a \in \mathcal{A} .
$$

Before proving this theorem we prove the following.

Lemma 3.4: If $C \in \mathcal{B}(\mathcal{K}, \mathcal{H})$ has dense range and $A, B \in \mathcal{B}(\mathcal{H})^{+}$, then, with the notation as in (A1) and (A4), we have

$$
\left(C^{*} A C\right):\left(C^{*} B C\right)=C^{*}(A: B) C \text { and }\left[C^{*} A C\right]\left(C^{*} B C\right)=C^{*}([A] B) C .
$$

Proof: Indeed, for arbitrary $k \in \mathcal{K}$, according to (A3),

$$
\left\langle C^{*}(A: B) C k, k\right\rangle=\langle(A: B) C k, C k\rangle=\inf \{\langle A f, f\rangle+\langle B(C k-f), C k-f\rangle \mid f \in \mathcal{H}\},
$$

and, taking into account that $C$ has dense range, this is

$$
\begin{aligned}
& =\inf \{\langle A C g, C g\rangle+\langle B(C k-C g), C k-C g\rangle \mid g \in \mathcal{K}\} \\
& =\inf \left\{\left\langle C^{*} A C g, g\right\rangle+\left\langle C^{*} B(C k-C g), k-g\right\rangle \mid g \in \mathcal{K}\right\} \\
& =\left\langle\left(C^{*} A C\right):\left(C^{*} B C\right) k, k\right\rangle .
\end{aligned}
$$

Then 
$\left[C^{*} A C\right]\left(C^{*} B C\right) k=\lim _{n \rightarrow \infty}\left(n C^{*} A C: C^{*} B C\right) k=\lim _{n \rightarrow \infty} C^{*}(n A: B) C k=C^{*} \lim _{n \rightarrow \infty}(n A: B) C k=C^{*}([A] B) C k$.

Proof of Theorem 3.3: We use the notation as in Sec. II. Let $\left(\pi_{\rho} ; \mathcal{K}_{\rho} ; V_{\rho}\right)$ be the minimal Stinespring representation for $\rho$. To simplify the notation, let

$$
A=D_{\rho}(\varphi), \quad B=D_{\rho}(\psi) .
$$

Clearly, the Radon--Nikodym derivative of $\theta=\varphi+\psi$ with respect to $\rho$ is $D_{\rho}(\varphi)+D_{\rho}(\psi)=A+B$. Let $\mathcal{K}_{\rho, 1}=\operatorname{Ker}(A+B)$ and $\mathcal{K}_{\rho, 2}=\mathcal{K}_{\rho} \ominus \operatorname{Ker}(A+B)$, subspaces in $\mathcal{K}_{\rho}$. With respect to the decomposition $\mathcal{K}=\mathcal{K}_{1} \oplus \mathcal{K}_{2}$ we have

$$
A+B=\left[\begin{array}{ll}
0 & 0 \\
0 & C
\end{array}\right], \quad \pi_{\rho}(a)=\left[\begin{array}{cc}
\pi_{\rho, 1}(a) & 0 \\
0 & \pi_{\rho, 2}(a)
\end{array}\right] \quad \text { for all } a \in \mathcal{A}, \quad \text { and } \quad V=\left[\begin{array}{l}
V_{1} \\
V_{2}
\end{array}\right],
$$

where $C$ is an operator in $\mathcal{B}\left(\mathcal{K}_{2}\right)^{+}$, necessarily one to one, and commutes with $\pi_{\rho, 2}(a)$, for all $a$ $\in \mathcal{A}$. By Theorem 2.9, the triple $\left(\pi_{\rho, 2} ; \mathcal{K}_{\rho, 2} ; C^{1 / 2} V_{\rho}\right)$ is the minimal Stinespring representation for $\theta=\varphi+\psi$. In order to simplify the notation, let $F=D_{\theta}(\psi)$, hence $I-F=D_{\theta}(\varphi)$ (see the proof of Theorem 3.1). Also, by Lemma 3.2 we have $D_{\theta}\left(\varphi_{\mathrm{ac}}\right)=[F](1-F)$. Then, for all $a \in \mathcal{A}$,

$$
\begin{aligned}
\psi(a) & =\left(C^{1 / 2} V_{\rho, 2}\right)^{*} F \pi_{\rho, 2}(a)\left(C^{1 / 2} V_{\rho, 2}\right)=V_{\rho, 2}^{*} C^{1 / 2} F C^{1 / 2} \pi_{\rho, 2}(a) V_{\rho, 2} \\
& =\left[\begin{array}{ll}
V_{1}^{*} & V_{\rho, 2}^{*}
\end{array}\right]\left[\begin{array}{cc}
0 & 0 \\
0 & C^{1 / 2} F C^{1 / 2}
\end{array}\right]\left[\begin{array}{cc}
\pi_{\rho, 1}(a) & 0 \\
0 & \pi_{\rho, 2}(a)
\end{array}\right]\left[\begin{array}{c}
V_{\rho, 1} \\
V_{\rho, 2}
\end{array}\right] \\
& =V_{\rho}^{*}\left[\begin{array}{cc}
0 & 0 \\
0 & C^{1 / 2} F C^{1 / 2}
\end{array}\right] \pi_{\rho}(a) V_{\rho} .
\end{aligned}
$$

In a similar way,

$$
\varphi(a)=V_{\rho}^{*}\left[\begin{array}{cc}
0 & 0 \\
0 & C^{1 / 2}(1-F) C^{1 / 2}
\end{array}\right] \pi_{\rho}(a) V_{\rho}
$$

and

$$
\varphi_{\mathrm{ac}}(a)=V_{\rho}^{*}\left[\begin{array}{cc}
0 & 0 \\
0 & C^{1 / 2}[F](1-F) C^{1 / 2}
\end{array}\right] \pi_{\rho}(a) V_{\rho} .
$$

By the uniqueness of the Radon-Nikodym derivative we obtain that

$$
A=\left[\begin{array}{cc}
0 & 0 \\
0 & C^{1 / 2} F C^{1 / 2}
\end{array}\right] \text { and } B=\left[\begin{array}{cc}
0 & 0 \\
0 & C^{1 / 2}(1-F) C^{1 / 2}
\end{array}\right] .
$$

Note that $C^{1 / 2}$ must also be one to one and hence, as a non-negative operator, it has dense range. Thus, by Lemma 3.4,

$$
\begin{aligned}
{[A] B } & =\left[\left[\begin{array}{cc}
0 & 0 \\
0 & C^{1 / 2} F C^{1 / 2}
\end{array}\right]\right]\left[\begin{array}{cc}
0 & 0 \\
0 & C^{1 / 2}(1-F) C^{1 / 2}
\end{array}\right]=\left[\begin{array}{cc}
0 & 0 \\
0 & {\left[C^{1 / 2} F C^{1 / 2}\right]\left(C^{1 / 2}(1-F) C^{1 / 2}\right)}
\end{array}\right] \\
& =\left[\begin{array}{cc}
0 & 0 \\
0 & C^{1 / 2}[F](1-F) C^{1 / 2}
\end{array}\right]=D_{\rho}\left(\varphi_{\mathrm{ac}}\right) .
\end{aligned}
$$

Since $\varphi-\varphi_{\mathrm{ac}}=\varphi_{s}$, we also have that $D_{\rho}\left(\varphi_{\mathrm{s}}\right)=B-[A] B$.

Finally, the formulas (3.6) and (3.7) can be obtained easily from (3.5), Theorem 6.4, Theorem 2.2, and Theorem 3.1.

The constructions of $\varphi_{\mathrm{ac}}$ and $\varphi_{s}$ performed during the proof of Theorem 3.1 can be given in terms of any $\theta=s \varphi+t \psi$, for $s, t>0$, with minor modifications, similar to those in (3.2), and, as a 
consequence of Theorem 3.3, they produce the same maximal Lebesgue decomposition. If both $\varphi$ and $\psi$ are unital, then $\theta=\frac{1}{2} \varphi+\frac{1}{2} \psi$ is a unital completely positive map as well. In this case, Parthasarthy ${ }^{28}$ gave the definitions as in (3.2) and the decomposition $\varphi=\varphi_{\mathrm{ac}}+\varphi_{\text {s }}$ was called the $\psi$ -Lebesgue decomposition of $\varphi$, while $\varphi_{\mathrm{ac}}$ and $\varphi_{s}$ were called the absolutely continuous part and, respectively, the singular part of $\varphi$ with respect to $\psi$. Further, in Ref. $28 \varphi$ was called $\psi$-absolutely continuous if $\varphi_{s}=0$. As a consequence of Theorems 3.1 and 3.3 it follows that this notion of absolute continuity is the same with that introduced in Sec. II C.

Corollary 3.5: Let $\varphi, \psi \in \mathrm{CP}(\mathcal{A} ; \mathcal{H})$.

(a) $\varphi$ is $\psi$-absolutely continuous if and only if $\varphi_{s}=0$.

(b) $\varphi$ is $\psi$-singular if and only if $\varphi_{\mathrm{ac}}=0$.

Another consequence of the results we got so far refers to other characterizations of singularity for completely positive maps. The equivalence of (iii)-(vi) is contained in Corollary 1.4.4 of Ref. 6.

Corollary 3.6: With the notation as before, the following assertions are equivalent.

(i) $\varphi$ is $\psi$-singular.

(ii) $\psi$ is $\varphi$-singular.

(iii) The Radon-Nikodym derivative $D_{\varphi+\psi}(\varphi)$ is a projection.

(iv) The Radon-Nikodym derivative $D_{\varphi+\psi}(\psi)$ is a projection.

(v) $\varphi$ is an extremal element of the convex set $\{\rho \in \operatorname{CP}(\mathcal{A} ; \mathcal{H}) \mid \rho \leq \varphi+\psi\}$.

(vi) $\quad \psi$ is an extremal element of the convex set $\{\rho \in \operatorname{CP}(\mathcal{A} ; \mathcal{H}) \mid \rho \leq \varphi+\psi\}$.

Proof: Due to the symmetry of the relation of singularity, it is sufficient to prove that (i), (iii), and (v) are mutually equivalent.

If $\varphi$ is $\psi$-singular, by the above corollary it follows that $\varphi=\varphi_{s}$ and hence, by (3.4) it follows that the Radon-Nikodym derivative $D_{\varphi+\psi}(\varphi)$ is a projection.

Conversely, if the Radon-Nikodym derivative $D_{\varphi+\psi}(\varphi)$ is a projection then, by (3.3), it follows that $D_{\theta}\left(\varphi_{\mathrm{ac}}\right)=0$ and hence $\varphi_{\mathrm{ac}}=0$, that is, $\varphi$ is $\psi$-absolutely continuous. Thus, (i) is equivalent with (iii).

In order to prove the equivalence of (iii) with (v), we use Theorem 2.2 and the well-known fact that, given a Hilbert space $\mathcal{H}$, the set of extremal elements of the $\operatorname{set}\{B \in \mathcal{B}(\mathcal{H}) \mid 0 \leq B \leq I\}$ coincides with the set of orthogonal projections in $\mathcal{H}$ (e.g., see Lemma 3.2 in Ref. 12).

In view of Proposition 2.7, another consequence of Theorem 3.1 is the following.

Corollary 3.7: Let $\varphi, \psi, \rho \in \operatorname{CP}(\mathcal{A} ; \mathcal{H})$ and consider the maximal $\rho$-Lebesgue decompositions $\varphi=\varphi_{\mathrm{ac}}+\varphi_{\mathrm{s}}$ and $\psi=\psi_{\mathrm{ac}}+\psi_{s}$.

(i) For any $t \geq 0$ we have $(t \psi)_{\mathrm{ac}}=t \psi_{\mathrm{ac}}$.

(ii) $\varphi_{\mathrm{ac}}+\psi_{\mathrm{ac}} \leq(\varphi+\psi)_{\mathrm{ac}}$.

(ii) If $\psi \leq \varphi$ then $\psi_{\mathrm{ac}} \leq \varphi_{\mathrm{ac}}$.

\section{B. Uniqueness}

The maximal $\psi$-Lebesgue decomposition $\varphi=\varphi_{\mathrm{ac}}+\varphi_{s}$ defined at (3.2) is unique by its maximality property but, if the condition of maximality is dropped, then the uniqueness may be affected. We examine first some sufficient conditions of uniqueness of Lebesgue decompositions.

\section{Proposition 3.8:}

(i) If $\varphi, \psi \in \mathrm{CP}(\mathcal{A} ; \mathcal{H})$ and $\varphi_{\mathrm{ac}} \leq_{\mathrm{u}} \psi$, that is, there exists $t>0$ such that $\varphi_{\mathrm{ac}} \leq t \psi$, then $\varphi$ admits a unique $\psi$-Lebesgue decomposition.

(ii) If $\psi \in \operatorname{CP}(\mathcal{A} ; \mathcal{H})$ is such that the property of $\psi$-absolute continuity is hereditary then, for any $\varphi \in \operatorname{CP}(\mathcal{A} ; \mathcal{H})$ there exists a unique $\psi$-Lebesgue decomposition of $\varphi$.

Proof: 
(i) Without loss of generality we can assume that $\varphi_{\mathrm{ac}} \leq \psi$. Consider the maximal $\psi$-Lebesgue decomposition of $\varphi=\varphi_{\mathrm{ac}}+\varphi_{s}$ as well as another $\psi$-Lebesgue decomposition $\varphi=\varphi_{1}+\varphi_{0}$, where $\varphi_{1}, \varphi_{0} \in \mathrm{CP}(\mathcal{A} ; \mathcal{H}), \varphi_{1}$ is $\psi$-absolutely continuous and $\varphi_{0}$ is $\psi$-singular. Then, taking into account the maximality of $\varphi_{\mathrm{ac}}$ it follows that $\varphi_{0}=\varphi-\varphi_{1} \geq \varphi_{\mathrm{ac}}-\varphi_{1} \geq 0$, hence $\varphi_{\mathrm{ac}}-\varphi_{1}$ is $\varphi$-singular. Since $\varphi_{\mathrm{ac}}-\varphi_{1}$ is $\psi$-absolutely continuous, it follows that $\varphi_{\mathrm{ac}}=\varphi_{1}$, and hence $\varphi_{\mathrm{s}}=\varphi_{0}$.

(ii) Similar to the proof of (i).

When comparing with the results on uniqueness of the Lebesgue decomposition in Appendix A 3, the above proposition looks very weak. The difficulty of transposing Theorem 6.5 and its Corollary 6.6 to the setting of completely positive maps comes from the fact that, in this case, the Radon-Nikodym derivatives live in the von Neumann algebra $\pi(\mathcal{A})^{\prime}$ that can be "very small." This is illustrated in the following.

Example 3.9: Let $\mathcal{A}$ be the $C^{*}$-algebra $L^{\infty}[0,1]$ and $\mathcal{H}=L^{2}[0,1]$. Let $\varphi, \psi: \mathcal{A} \rightarrow \mathcal{B}(\mathcal{H})$ be defined by $(\varphi(f) g)(g)=t f(t) g(t)$ and $(\psi(f) g)(g)=(1-t) f(t) g(t)$, for $f \in L^{\infty}[0,1]$ and $g \in L^{2}[0,1]$. Then both $\varphi$ and $\psi$ are completely positive and $\varphi+\psi=\theta$ is the map $(\theta(f) g)(t)=f(t) g(t)$, for $f$ $\in L^{\infty}[0,1]$ and $g \in L^{2}[0,1]$. Then the minimal Stinespring representation of $\theta$ is $\left(\varphi_{\theta} ; \mathcal{K}_{\theta} ; V_{\theta}\right)$ where, $\mathcal{K}_{\theta}=L^{2}[0,1], \pi_{\theta}(f)=M_{f}$, the operator of multiplication with $f \in L^{\infty}[0,1]$, and $V_{\theta}$ is the identity operator on $L^{2}[0,1]$. We have that $\pi_{\theta}(\mathcal{A})^{\prime}=\left\{M_{f} \mid f \in L^{\infty}[0,1]\right\}$ is a maximal Abelian von Neumann algebra in $\mathcal{B}\left(L^{2}[0,1]\right)$. On the other hand, $\psi$ is $\varphi$-absolutely continuous and there exists only one Lebesgue decomposition of $\psi$ with respect to $\varphi$. However, there exists no $k$ such that $\psi \leq k \varphi$.

For the case of $\mathcal{H}=\mathrm{C}$, the difficulties on the uniqueness, and an example of nonuniqueness, see Kosaki. $^{22}$

Finally, we observe that when considering the complete isomorphism $\mathrm{CP}(\mathrm{C} ; \mathcal{H}) \ni \varphi \mapsto \varphi(1)$ $\in \mathcal{B}(\mathcal{H})^{+}$, as explained at the end of Sec. III A, an application of Theorem 6.5 gives the nonuniqueness of the Lebesgue decomposition for some operator valued completely positive maps.

\section{AN APPLICATION TO THE INFIMUM PROBLEM FOR QUANTUM OPERATIONS}

Let $\mathcal{H}$ be a Hilbert space and $\mathcal{B}(\mathcal{H})^{+}$the cone of bounded non-negative operators in $\mathcal{H}$. Given $A, B \in \mathcal{B}(\mathcal{H})^{+}$, the infimum of $A$ and $B$, denoted by $A \wedge B$, is the greatest lower bound of the set $\{A, B\}$ in the ordered set $\mathcal{B}(\mathcal{H})^{+}$, that is, $A \wedge B \leq A, B$ and, if $C \in \mathcal{B}(\mathcal{H})^{+}$is such that $C \leq A, B$, then $C \leq A \wedge B$. Questions on characterization of the existence and computation of the infimum operator are related to the lattice properties of quantum effects, cf. Gudder. ${ }^{17}$ First, let us note that $B$ is $A$-singular if and only if $A \wedge B=0$, hence, it is expected that this kind of questions should be related with the Radon-Nikodym derivatives and Lebesgue decompositions. The complete answer to this question, when considering only quantum effects, is given by the following theorem. For the notation on the shorted operator $[A] B$ see Appendix A.

Theorem 4.1: [Ando (Ref. 4)] Let $A, B \in \mathcal{B}(\mathcal{H})^{+}$. Then $A \wedge B$ exists if and only if the RadonNikodym derivatives $[A] B$ and $[B] A$ are comparable, that is, either $[A] B \leq[B] A$ or $[B] A \leq[A] B$. In this case, $A \wedge B=\min \{[A] B,[B] A\}$.

For other (more restrictive) formulas of calculating the infimum $A \wedge B$, see also Ref. 15 and the bibliography cited there.

When considering open quantum systems, quantum operations take the place of the quantum effects, and in connection with their lattice properties the infimum problem for quantum operations can be stated in similar terms: for $\varphi, \psi \in \mathrm{CP}(\mathcal{A} ; \mathcal{H})$ we write $\varphi \wedge \psi \in \operatorname{CP}(\mathcal{A} ; \mathcal{H})$ (if it exists) for the infimum of $\varphi$ and $\psi$, that is, $\varphi \wedge \psi \leq \varphi, \psi$ and for any $\rho \in \operatorname{CP}(\mathcal{A} ; \mathcal{H})$ such that $\rho \leq \varphi, \psi$, it follows $\rho \leq \varphi \wedge \psi$. With this definition, $\varphi$ is $\psi$-singular if and only if $\varphi \wedge \psi=0$. As an application of our results on the Lebesgue decomposition, we can show that the picture for quantum operations is similar to that for quantum effects.

Theorem 4.2: Let $\varphi, \psi \in \operatorname{CP}(\mathcal{A} ; \mathcal{H})$ and consider $\varphi=\varphi_{\mathrm{ac}}+\varphi_{s}$, the $\psi$-Lebesgue decomposition of $\varphi$, as well as $\psi=\psi_{\mathrm{ac}}+\psi_{s}$, the $\varphi$-Lebesgue decomposition of $\psi$. Then the infimum $\varphi \wedge \psi$ exists if 
and only if $\varphi_{\mathrm{ac}}$ and $\psi_{\mathrm{ac}}$ are comparable and, in this case,

$$
\varphi \wedge \psi=\min \left\{\varphi_{\mathrm{ac}}, \psi_{\mathrm{ac}}\right\}
$$

We need an auxiliary result.

Lemma 4.3: Let $A \in \mathcal{B}(\mathcal{H})^{+}$be a contraction and let $\mathcal{M}$ be a von Neumann algebra in $\mathcal{B}(\mathcal{H})$ such that $A \in \mathcal{M}$. Then the following are equivalent.

(i) The infimum of $A$ and $I-A$ with respect to $\mathcal{M}^{+}$exists.

(ii) The infimum of $A$ and $I-A$ with respect to $\mathcal{B}(\mathcal{H})^{+}$exists.

(iii) $\sigma(A)$, the spectrum of $A$, is contained either in $\{0\} \cup\left[\frac{1}{2}, 1\right]$ or in $\left[0, \frac{1}{2}\right] \cup\{1\}$.

Moreover, in the case when any of the assertions (i), (ii), or (iii) holds, then the two infima at (i) and, respectively, (ii) coincide and, letting $g \in C([0,1])$ be the function,

$$
g(t)=\min \{t, 1-t\}= \begin{cases}t, & 0 \leq t \leq 1 / 2, \\ 1-t, & 1 / 2, \leq t \leq 1,\end{cases}
$$

we have, by continuous functional calculus, $A \wedge(I-A)=g(A)$.

Proof: The equivalence of (ii) and (iii) is proven in Theorem 3.1 in Ref. 15. Clearly (ii) implies (i).

In order to prove (i) implies (iii), we observe that the proof of the corresponding implication (ii) implies (iii) in Theorem 3.1 in Ref. 15 is done in such a way that all the constructions are kept within the von Neumann algebra generated by $A$, and hence in $\mathcal{M}$.

Proof of Theorem 4.2: Let $\theta=\varphi+\psi$ and consider the Radon-Nikodym derivatives $D_{\theta}(\varphi)$ and $D_{\theta}(\psi)$, as in the proof of Theorem 3.1. Then (3.1) holds. By Theorem 2.2, it follows that $\varphi \wedge \psi$ exists in $\operatorname{CP}(\mathcal{A} ; \mathcal{H})$ if and only if $D_{\theta}(\varphi) \wedge D_{\theta}(\psi)$ exists with respect to the von Neumann algebra $\pi(\mathcal{A})^{\prime}$. By Lemma 4.3, it follows that the latter is equivalent with the fact that $D_{\theta}(\varphi) \wedge D_{\theta}(\psi)$ exists with respect to $\mathcal{B}(\mathcal{H})^{+}$and then, by Theorem 4.1, this is equivalent with the fact that the shorted operators $\left[D_{\theta}(\varphi)\right] D_{\theta}(\psi)$ and $\left[D_{\theta}(\psi)\right] D_{\theta}(\varphi)$ are comparable. But, in view of (3.3), these shorted operator are, respectively, $D_{\theta}\left(\psi_{\mathrm{ac}}\right)$ and $D_{\theta}\left(\psi_{\mathrm{ac}}\right)$. Using once again Theorem 2.2, it follows that $\varphi \wedge \psi$ exists if and only if $D_{\theta}\left(\psi_{\mathrm{ac}}\right)$ and $D_{\theta}\left(\psi_{\mathrm{ac}}\right)$ are comparable, and (4.1) holds.

Let us observe that, by the assertion (iii) in Lemma 4.3 and with the notation as in the preceding proof, a formula for computing $\varphi \wedge \psi$ that is more explicit than (4.1), can be given in terms of the infimum of the Radon-Nikodym derivatives $D_{\theta}(\varphi) \wedge D_{\theta}(\psi)$.

\section{THE LEBESGUE DECOMPOSITION FOR COMPLETELY POSITIVE MAPS ON MATRICES}

In this section we focus on completely positive maps from $M_{n}$, the $C^{*}$-algebra of $n \times n$ matrices, to $M_{k}$, for which we describe the Lebesgue decomposition in terms of the Choi matrices. This situation corresponds to applications to quantum information theory in the sense that in order to make the Lebesgue-Radon-Nikodym decomposition available to quantum information theorists this should be described in terms of the Choi's matrix, cf. Leung. ${ }^{25}$ In this final dimensional case an additional and very helpful fact is that there exists a completely positive map that uniformly majorizes all the others, namely, the tracial completely positive map. To this end, we first recall, in a slightly different formulation, known results on the structure of completely positive maps, cf. $^{11,7,24,31}$ and the bibliography cited there. Because of this, we will skip most of the proofs.

First note that, in this finite dimensional case, there exists only one Lebesgue decomposition, as explained in Sec. III B.

For $n \in \mathbb{N}$ let $\left\{e_{i}^{(n)}\right\}_{i=1}^{n}$ be the canonical basis of $\mathbb{C}^{n}$. For $n, k \in \mathbb{N}$ we consider the matrix units $\left\{E_{i, j}^{(n, k)} \mid i=1, \ldots, n, j=1, \ldots, k\right\} \subset M_{n, k}$ of size $n \times k$ (as usually, the space $M_{n, k}$ of $n \times k$ matrices is identified with $\left.\mathcal{B}\left(\mathrm{C}^{k}, \mathrm{C}^{n}\right)\right)$, that is, $E_{i, j}^{(n, k)}$ is the $n \times k$ matrix with all entries 0 except the $(i, j)$ th entry which is 1 . In case $n=k$, we denote simply $E_{i, j}^{(n)}=E_{i, j}^{(n, n)}$. 
Here and in the following we use the tensor notation for rank one operators, that is, if $\mathcal{H}$ and $\mathcal{K}$ are Hilbert spaces and $h \in \mathcal{H}$ and $k \in \mathcal{K}$ are nontrivial vectors, then the rank 1 operator $h \otimes \bar{k}$ $\in \mathcal{B}(\mathcal{K}, \mathcal{H})$ is defined by $(h \otimes \bar{k}) x=\langle x, k\rangle h$ for all $x \in \mathcal{H}$. With this notation we have

$$
E_{i, j}^{(n, k)}=e_{i}^{(n)} \otimes \bar{e}_{j}^{(k)}, \quad i=1, \ldots, n, \quad j=1, \ldots, k .
$$

We also record the following direct consequences of the definitions: for all $j=1, \ldots, n$ and $i$ $=1, \ldots, k$ we have

$$
E_{i, j}^{(n, k)^{*}}=E_{j, i}^{(k, n)}
$$

and if, in addition, $p \in \mathbb{N}, r=1, \ldots, k$, and $s=1, \ldots, p$, then

$$
E_{i, j}^{(n, k)} E_{r, s}^{(k, p)}=\delta_{j, r} E_{i, s}^{(n, p)} .
$$

We also use the lexicographic reindexing of $\left\{E_{i, j}^{(n, k)} \mid i=1, \ldots, n, j=1, \ldots, k\right\}$, more precisely

$$
\left(E_{1,1}^{(n, k)}, \ldots, E_{1, k}^{(n, k)}, E_{2,1}^{(n, k)}, \ldots, E_{2, k}^{(n, k)}, \ldots, E_{n, 1}^{(n, k)}, \ldots, E_{n, k}^{(n, k)}\right)=\left(\mathcal{E}_{1}, \mathcal{E}_{2}, \ldots, \mathcal{E}_{n k}\right) .
$$

An even more explicit form of this reindexing is the following:

$$
\mathcal{E}_{r}=E_{i, j}^{(n, k)} \text { where } r=(j-1) k+i, \quad \text { for all } i=1, \ldots, n, \quad j=1, \ldots, k .
$$

Lemma 5.1: The formula

$$
\varphi_{(m-1) k+i,(l-1) k+j}=\left\langle\varphi\left(E_{i, j}^{(n)}\right) e_{l}^{(k)}, e_{m}^{(k)}\right\rangle, \quad m, l=1, \ldots, k, \quad i, j=1, \ldots, n,
$$

and its inverse

$$
\varphi(C)=\sum_{r, s}^{n k} \varphi_{r, s} \mathcal{E}_{r}^{*} C \mathcal{E}_{s}, \quad C \in M_{n}
$$

establish a linear and bijective correspondence,

$$
\mathcal{B}\left(M_{n}, M_{k}\right) \ni \varphi \mapsto \Phi=\left[\varphi_{r, s}\right]_{r, s=1}^{n k} \in M_{n k}
$$

Given $\varphi \in \mathcal{B}\left(M_{n}, M_{k}\right)$ the matrix $\Phi$ as in (5.8) is called the Choi matrix of $\varphi$.

Remark 5.2: With respect to the identification $\mathbb{C}^{n k} \simeq \mathbb{C}^{n} \otimes \mathbb{C}^{k}$, any matrix $\Phi=\left[\varphi_{r, s}\right]_{r, s=1}^{n k} \in M_{n k}$ $=\mathcal{B}\left(\mathrm{C}^{n k}\right)$ is identified with a linear operator $\Phi \in \mathcal{B}\left(\mathbb{C}^{n} \otimes \mathbb{C}^{k}\right)$, in such a way that the formula (5.6) becomes

$$
\varphi_{(m-1) k+i,(l-1) k+j}=\left\langle\Phi\left(e_{j}^{(n)}\right) \otimes e_{l}^{(k)}, e_{i}^{(n)} \otimes e_{m}^{(k)}\right\rangle, \quad m, l=1, \ldots, k, \quad i, j=1, \ldots, n .
$$

Remark 5.3: In the correspondence in Lemma 5.1, $\varphi$ is unital if and only if

$$
\sum_{i=1}^{n} \varphi_{(m-1) k+i,(l-1) m+i}=\delta_{l, m} \text { for all } l, m \in\{1, \ldots, k\} .
$$

In the following, to a certain extent, our arguments and calculations will be parallel to those performed by Raginsky in Sec. V.A of Ref. 31 but we will go further in exploiting the explicit formulas for the Lebesgue decomposition obtained in Theorem 3.3.

Let $\rho: M_{n} \rightarrow M_{k}$ be the linear map defined by

$$
\rho(C)=\frac{1}{n} \operatorname{tr}(C) I_{k}, \quad C \in M_{n} .
$$

Let the linear mapping 


$$
V: \mathbb{C}^{k} \rightarrow \mathbb{C}^{n^{2} k} \simeq \mathbb{C}^{n} \otimes \mathbb{C}^{n k} \simeq \mathbb{C}^{n} \otimes \mathbb{C}^{n} \otimes \mathbb{C}^{k}
$$

be defined by

$$
V h=\frac{1}{\sqrt{n}}\left[\begin{array}{c}
\mathcal{E}_{1} h \\
\mathcal{E}_{2} h \\
\vdots \\
\mathcal{E}_{n k} h
\end{array}\right], \quad h \in \mathbb{C}^{k},
$$

or, equivalently, with the identification $\mathbb{C}^{n^{2} k} \simeq \mathrm{C}^{n} \otimes \mathrm{C}^{n} \otimes \mathrm{C}^{k}$ and the reindexing defined at (5.4),

$$
V h=\sum_{i=1}^{n} \sum_{j=1}^{k} E_{i, j}^{(n, k)} h \otimes e_{i}^{(n)} \otimes e_{j}^{(k)} .
$$

We consider also the map

$$
\pi: M_{n} \rightarrow M_{n^{2} k} \simeq \mathcal{B}\left(\mathbb{C}^{n^{2} k}\right) \simeq \mathcal{B}\left(\mathbb{C}^{n} \otimes \mathbb{C}^{n k}\right),
$$

defined by

$$
\pi(C)=C \otimes I_{n k}, \quad C \in M_{n} .
$$

Lemma 5.4: With the notation as in $(5.10)-(5.13),\left(\pi ; \mathbb{C}^{n^{2} k} ; V\right)$ is the minimal Stinespring representation of $\rho$, in particular, $\rho \in \mathrm{CP}\left(M_{n}, M_{k}\right)$.

In addition, $\rho$ uniformly dominates any map $\varphi \in \mathrm{CP}\left(M_{n}, M_{k}\right)$.

Lemma 5.4 can be used to obtain the following result of $\operatorname{Kraus}^{23}$ and Choi: ${ }^{11}$ Let $\varphi: M_{n}$ $\rightarrow M_{k}$ be a completely positive map. Then there are $n \times k$ matrices $V_{1}, V_{2}, \ldots, V_{m}$ with $m \leq n k$ such that

$$
\varphi(A)=V_{1}^{*} A V_{1}+V_{2}^{*} A V_{2}+\cdots+V_{m}^{*} A V_{m} \text { for all } A \in M_{n} .
$$

We also record the following fact: if $\mathcal{H}$ and $\mathcal{K}$ are Hilbert spaces and $\Phi, \Psi \in \mathcal{B}(\mathcal{K})^{+}$, then it is easy to see, from (A2) and (A4), that

$$
\left(I_{\mathcal{H}} \otimes \Phi\right):\left(I_{\mathcal{H}} \otimes \Psi\right)=I_{\mathcal{H}} \otimes(\Phi: \Psi), \quad\left[I_{\mathcal{H}} \otimes \Phi\right]\left(I_{\mathcal{H}} \otimes \Psi\right)=I_{\mathcal{H}} \otimes[\Phi] \Psi .
$$

The main result of this section is the following description of the Lebesgue decomposition for completely positive maps between matrices, in terms of Choi matrices. For the definition of the "shorted matrix" $[\Psi] \Phi$, see Appendix A.

Theorem 5.5: The formula (5.6) and its inverse (5.7) establish an affine and order-preserving isomorphism,

$$
\mathrm{CP}\left(M_{n}, M_{k}\right) \ni \varphi \mapsto \Phi \in M_{n k}^{+} .
$$

Moreover, if $\varphi, \psi \in \mathrm{CP}\left(M_{n}, M_{k}\right), \Phi, \Psi \in M_{n k}^{+}$are the positive $n k \times n k$ matrices corresponding by (5.8) to $\varphi$ and, respectively, $\psi$, and the positive matrices $A, B \in M_{n k}$ are defined by

$$
A=\left[\alpha_{i, j}\right]_{i, j=1}^{n k}=[\Psi] \Phi, \quad B=\left[\beta_{i, j}\right]_{i, j=1}^{n k}=\Phi-[\Psi] \Phi,
$$

then, the absolutely continuous part $\varphi_{\mathrm{ac}}$ and the singular part $\varphi_{s}$ of $\varphi$ with respect to $\psi$ are given by the following formulas:

$$
\varphi_{\mathrm{ac}}(C)=\sum_{r, s=1}^{n k} \alpha_{r, s} \mathcal{E}_{r}^{*} C \mathcal{E}_{s}, \quad \varphi_{\mathrm{s}}(C)=\sum_{r, s=1}^{n k} \beta_{r, s} \mathcal{E}_{r}^{*} C \mathcal{E}_{s}, \quad \text { for all } C \in M_{n} .
$$

Proof: Consider the completely positive map $\rho: M_{n} \rightarrow M_{k}$ defined at (5.10), as well as its 
minimal Stinespring representation $\left(\pi ; \mathrm{C}^{n^{2} k} ; V\right)$, as proven in Lemma 5.4. We combine the facts contained in Lemma 5.1 and Lemma 5.4 with those in Corollary 2.3 in order to get that the Radon-Nikodym derivative with respect to $\rho$ establishes an affine and order-preserving isomorphism between the cones,

$$
\mathrm{CP}\left(M_{n}, M_{k}\right) \ni \varphi \mapsto D_{\rho}(\varphi) \in \pi\left(M_{n}\right)^{\prime+} .
$$

Since

$$
\pi\left(M_{n}\right)^{\prime}=\left(M_{n} \otimes I_{n k}\right)^{\prime}=I_{n} \otimes M_{n k},
$$

and this identification induces an affine and order-preserving isomorphism between the corresponding cones of positive elements, it follows that the Radon-Nikodym derivative with respect to $\rho$ establishes an affine and order-preserving isomorphis,

$$
\mathrm{CP}\left(M_{n}, M_{k}\right) \ni \varphi \mapsto \Phi \in M_{n k}^{+},
$$

more precisely

$$
D_{\rho}(\varphi)=I_{n} \otimes \Phi, \quad \varphi \in \mathrm{CP}\left(M_{n}, M_{k}\right) .
$$

It remains to prove that the isomorphism (5.20) coincides with that defined at (5.16), which, by the uniqueness of the Radon-Nikodym derivative, is equivalent with proving that

$$
\varphi(C)=V^{*}\left(I_{n} \otimes \Phi\right)\left(C \otimes I_{n k}\right) V=V^{*}(C \otimes \Phi) V .
$$

To see this, it is sufficient to prove that for all $i, j \in\{1, \ldots, n\}$ and all $l, m \in\{1, \ldots, k\}$ we have

$$
\left\langle\varphi\left(E_{i, j}^{(n)} e_{l}^{(k)}, e_{m}^{(k)}\right\rangle=\left\langle V^{*}\left(E_{i, j}^{(n)} \otimes \Phi\right) V e_{l}^{(k)}, e_{m}^{(k)}\right\rangle .\right.
$$

First, we note that

$$
V e_{l}^{(k)}=\sum_{r=1}^{n} \sum_{s=1}^{k}\left(E_{r, s}^{(n) k} e_{l}^{(k)}\right) \otimes e_{r}^{(n)} \otimes e_{s}^{(k)}=\sum_{r=1}^{n} \sum_{s=1}^{k} \delta_{l, s} e_{r}^{(n)} \otimes e_{r}^{(n)} \otimes e_{s}^{(k)}=\sum_{r=1}^{n} e_{r}^{(n)} \otimes e_{r}^{(n)} \otimes e_{l}^{(k)}
$$

Then

$$
\begin{aligned}
\left\langle V^{*}\left(E_{i, j}^{(n)} \otimes \Phi\right) V e_{l}^{(k)}, e_{m}^{(k)}\right\rangle= & \left\langle\left(E_{i, j}^{(n)} \otimes \Phi\right) V e_{l}^{(k)}, V e_{m}^{(k)}\right\rangle=\sum_{r=1}^{n} \sum_{p=1}^{n}\left\langle\left(E_{i, j}^{(n)} \otimes \Phi\right)\left(e_{r}^{(n)} \otimes e_{r}^{(n)}\right) \otimes e_{l}^{(k)}, e_{p}^{(n)} \otimes e_{p}^{(n)}\right. \\
& \left.\otimes e_{m}^{(k)}\right\rangle=\sum_{r=1}^{n} \sum_{p=1}^{n}\left\langle\delta_{r, j} e_{i}^{(n)} \otimes\left(e_{r}^{(n)} \otimes e_{l}^{(k)}\right), e_{p}^{(n)} \otimes e_{p}^{(n)} \otimes e_{m}^{(k)}\right\rangle=\sum_{p=1}^{n}\left\langle e_{i}^{(n)}\right. \\
& \otimes\left(\Phi\left(e_{j}^{(n)} \otimes e_{l}^{(n)}\right), e_{p}^{(n)} \otimes e_{p}^{(n)} \otimes e_{m}^{(k)}\right\rangle=\sum_{p=1}^{n}\left\langle e_{i}^{(n)}, e_{p}^{(n)}\right\rangle\left\langle\left(\Phi \left( e_{j}^{(n)} \otimes e_{l}^{(n)}, e_{p}^{(n)}\right.\right.\right. \\
& \left.\otimes e_{p}^{(n)} \otimes e_{m}^{(k)}\right\rangle=\sum_{p=1}^{n} \delta_{j, p}\left\langle\left(\Phi\left(e_{j}^{(n)} \otimes e_{l}^{(n)}, e_{p}^{(n)} \otimes e_{p}^{(n)} \otimes e_{m}^{(k)}\right\rangle=\left\langle\Phi \left( e_{j}^{(n)}\right.\right.\right.\right. \\
& \left.\otimes e_{l}^{(k)}, e_{i}^{(n)} \otimes e_{m}^{(k)}\right\rangle=\left\langle\varphi\left(E_{i, j}^{(n)}\right) e_{l}^{(k)}, e_{m}^{(k)}\right\rangle,
\end{aligned}
$$

where, at the last step, we used (5.9) and (5.6). Thus, (5.22) is proven, and hence (5.21) is proven.

Finally, in order the get the second part of the statement, we use (5.15) and (5.20) and Theorem 3.3. 


\section{ACKNOWLEDGMENTS}

The first named author's research was partially supported from Grant No. 2-CEX-06-11-34/ 2006 from the Ministry of Education and Research. Also, part of this research was done during July and August 2007 when the first named author visited the Department of Mathematics and Statistics of the University of Calgary, courtesy of Paul Binding for whose hospitality the authors are warmly thankful.

\section{APPENDIX A: LEBESGUE DECOMPOSITIONS OF POSITIVE OPERATORS}

In this section we review the Radon-Nikodym derivatives and Lebesgue decompositions for bounded non-negative operators on a Hilbert space.

\section{Parallel sum}

Let $\mathcal{H}$ be a Hilbert space and let $\mathcal{B}(\mathcal{H})$ denote the $C^{*}$-algebra of bounded linear operators on $\mathcal{H}$. If $A, B \in \mathcal{B}(\mathcal{H})$ are self-adjoint we write $A \leq B$ if $\langle A h, h\rangle \leq\langle B h, h\rangle$ for all $h \in \mathcal{H}$, the natural order relation (reflexive, antisymmetric, and transitive). We also denote by $\mathcal{B}(\mathcal{H})^{+}$the convex strict cone of non-negative operators

Given $A, B \in \mathcal{B}(\mathcal{H})^{+}$the parallel sum of $A$ and $B$ (originally defined by Anderson and Duffin ${ }^{1}$ for matrices and then extended to bounded positive operators by Fillmore and Williams ${ }^{13}$ ) is

$$
A: B=A^{1 / 2} C^{*} D B^{1 / 2},
$$

where $C$ and $D$ are the minimal bounded operators that produce the factorizations $A^{1 / 2}=(A$ $+B)^{1 / 2} C$ and $B^{1 / 2}=(A+B)^{1 / 2} D$ (in fact, $C$ and $D$ can be characterized as certain pseudoinverses). The following formula holds:

$$
A: B=\mathrm{SO}-\lim _{\epsilon \backslash \infty}\left((A+\epsilon I)^{-1}+(B+\epsilon I)^{-1}\right)^{-1},
$$

where SO means that the limit should be taken with respect to the strong operator topology. Ando, ${ }^{3}$ and independently Pekarev and Shmulyan, ${ }^{30}$ obtained probably the most versatile formula,

$$
\langle(A: B) h, h\rangle=\inf \{\langle A g, g\rangle+\langle B(h-g), h-g\rangle \mid g \in \mathcal{H}\}, \quad h \in \mathcal{H} .
$$

Note that this binary operation is symmetric, more precisely, $A: B=B: A$, separately nondecreasing with respect to each argument, that is, if $A_{1} \leq A_{2}$ then $A_{1}: B \leq A_{2}: B$, and that $0 \leq A: B \leq A, B$, but it is not (separately) additive.

\section{Shorted operators as Radon-Nikodym derivatives}

Given $A, B \in \mathcal{B}(\mathcal{H})^{+}$, the shorted operator $[A] B \in \mathcal{B}(\mathcal{H})^{+}$is by definition (cf. Ando, ${ }^{3}$ who generalized the previous definition introduced by Anderson and Trapp ${ }^{2}$ ),

$$
([A] B) h:=\mathrm{SO}-\lim _{n \rightarrow \infty}(n A): B,
$$

where the SO limit exists because $(n A): B \leq B$ and $(n A): B \leq((n+1) A): B$ for all $n \in \mathbb{N}$. Note that $[A] B \leq B$ but $[A] B$ may not be comparable with $A$.

Another formula to calculate $[A] B$ is available. Namely, let $P_{A, B}$ denote the orthogonal projection of $\mathcal{H}$ onto the closure of the subspace $\left\{h \in \mathcal{H} \mid B^{1 / 2} h \in \operatorname{Ran}\left(A^{1 / 2}\right)\right\}$, where $\operatorname{Ran}(C)$ denotes the range of the operator $C$.

Theorem 6.1: [Kosaki (Ref. 21)] If $A, B \in \mathcal{B}(\mathcal{H})^{+}$then $[A] B=B^{1 / 2} P_{A, B} B^{1 / 2}$.

For given $A, B \in \mathcal{B}(\mathcal{H})^{+}$one says that $A$ uniformly dominates $B$, in brief $B \leq_{u} A$, if any of the following equivalent conditions holds.

(i) There exists $t>0$ such that $B \leq t A$, that is, $\langle B h, h\rangle \leq t\langle A h, h\rangle$ for all $h \in \mathcal{H}$.

(ii) $\quad \operatorname{Ran}\left(B^{1 / 2}\right) \subseteq \operatorname{Ran}\left(A^{1 / 2}\right)$. 
(iii) There exists $X \in \mathcal{B}(\mathcal{H})$ such that $B^{1 / 2}=A^{1 / 2} X$.

This is a partial preorder relation (only reflexive and transitive) on $\mathcal{B}(\mathcal{H})^{+}$.

Theorem 6.2: [Ando (Ref. 3)] Given $A, B \in \mathcal{B}(\mathcal{H})^{+}$, the following assertions are equivalent:

(a) There exists a sequence $\left(B_{n}\right)$ in $\mathcal{B}(\mathcal{H})^{+}$subject to the following conditions.

(aco1) $\left(B_{n}\right)$ is nondecreasing, in the sense that $B_{1} \leq B_{2} \leq \cdots \leq B_{n} \leq B_{n+1} \leq \cdots$.

(aco2) SO- $\lim _{n \rightarrow \infty} B_{n}=B$.

(aco3) For all $n \in \mathbb{N}, B_{n} \leq_{\mathrm{u}} A$ (i.e., for every $n \in \mathbb{N}$ there exists $t_{n}>0$ such that $B_{n} \leq t_{n} A$ ).

(b) $B=[A] B$, that is, SO- $\lim _{n \rightarrow \infty}(n A): B=B$.

(c) The linear manifold $\left\{h \in \mathcal{H} \mid B^{1 / 2} h \in \operatorname{Ran}\left(A^{1 / 2}\right)\right\}$ is dense in $\mathcal{H}$.

For given $A, B \in \mathcal{B}(\mathcal{H})^{+}$one says that $B$ is $A$-absolutely continuous, and we write $B \ll A$, if any of the equivalent assertions (a), (b), or (c) in Theorem 6.2 holds. The $A$-absolute continuity is additive in the sense that, if $B$ and $C$ are $A$-absolutely continuous then $B+C$ is $A$-absolutely continuous, but, in general, it is not hereditary, in particular, it is not a transitive relation. Clearly, if $B \leq{ }_{u} A$ then $B \ll A$. For the converse implication, the following result, that is implicit in Ref. 3 , holds.

Proposition 6.3: Let $A \in \mathcal{B}(\mathcal{H})^{+}$. The following assertions are equivalent.

(i) $\operatorname{Ran}(A)$ is closed.

(ii) For arbitrary $B \in \mathcal{B}(\mathcal{H})^{+}, B \leq_{u} A$ if and only if $B \ll A$.

Because of Theorem 6.2, and in analogy with the comparison theory for scalar valued positive measures, it is natural to call $[A] B$ the Radon-Nikodym derivative of $B$ with respect to $A$.

\section{Lebesgue decompositions}

In this subsection we review results obtained by $A$ ndo. ${ }^{3}$ If $A, C \in \mathcal{B}(\mathcal{H})^{+}$, one says that $C$ is $A$ -singular if the only operator $D \in \mathcal{B}(\mathcal{H})^{+}$satisfying $D \leq A$ and $D \leq C$ is $D=0$. Clearly, $C$ is $A$-singular if and only if $A$ is $C$-singular, so in this case we can call $A$ and $C$ mutually singular.

Given $A, B \in \mathcal{B}(\mathcal{H})^{+}$, a decomposition $B=B_{1}+B_{0}$ such that $B_{0}, B_{1} \in \mathcal{B}(\mathcal{H})^{+}, B_{1}$ is $A$-absolutely continuous and $B_{0}$ is $A$-singular, is called an $A$-Lebesgue decomposition of $B$.

Theorem 6.4: If $A, B \in \mathcal{B}(\mathcal{H})^{+}$then the decomposition

$$
B=[A] B+(B-[A] B)
$$

has the following two properties.

(i) $\quad[A] B$ is A-absolutely continuous and $B-[A] B$ is A-singular.

(ii) $\quad[A] B$ is the maximum of all $A$-absolutely continuous operators $C \in \mathcal{B}(\mathcal{H})^{+}$such that $C$ $\leq B$.

The Lebesgue-type decomposition in Theorem 6.4 is unique due to its maximality property but, in general, Lebesgue-type decompositions for positive operators are not unique.

Theorem 6.5: Given $A, B \in \mathcal{B}(\mathcal{H})^{+}, B$ admits a unique A-Lebesgue decomposition if and only if $[A] B \leq{ }_{u} A$, that is, there exists $t>0$ such that $[A] B \leq t A$.

Corollary 6.6: Given $A \in \mathcal{B}(\mathcal{H})^{+}$, the following assertions are equivalent.

(a) $\operatorname{Ran}(A)$ is closed.

(b) A-absolute continuity is a hereditary property.

(c) Any operator $B \in \mathcal{B}(\mathcal{H})^{+}$has a unique A-Lebesgue decomposition. 


\section{APPENDIX B: ABSOLUTE CONTINUITY FOR NON-NEGATIVE QUADRATIC FORMS}

In this Appendix we consider a comparison theory for quadratic forms on general (complex) vector spaces. This way actually puts the comparison theory of completely positive maps in a more natural context and offers an efficient framework to deal with approximation properties (e.g., in spectral sense) and establish connections with the existing theories of canonical forms considered by Simon $^{33}$ and Jørgensen. ${ }^{19}$

\section{Radon-Nikodym derivatives for non-negative quadratic forms}

Let $\mathcal{V}$ be a complex vector space. For a non-negative (that is, positive semidefinite) Hermitian form $p: \mathcal{V} \times \mathcal{V} \rightarrow \mathrm{C}$, recall that the Schwarz Inequality holds,

$$
|p(u, v)|^{2} \leq p(u, u) p(v, v), \quad u, v \in \mathcal{V},
$$

and, consequently,

$$
\operatorname{Ker}(p)=\{u \in \mathcal{V} \mid p(u, u)=0\}=\{u \in \mathcal{V} \mid p(u, v)=0 \text { for all } v \in \mathcal{V}\} .
$$

The polarization formula

$$
p(u, v)=\frac{1}{4} \sum_{k=0}^{3} \mathrm{i}^{k} p\left(u+\mathrm{i}^{k} v, u+\mathrm{i}^{k} v\right), \quad u, v \in \mathcal{V},
$$

shows that $p$ is uniquely determined by the quadratic form $\mathcal{V} \ni u \mapsto p(u)=p(u, u)$. Thus, one uses freely the notion of non-negative quadratic form instead of the notion of positive semidefinite Hermitian form.

A pair $(\mathcal{K} ; \Pi)$ is called a Hilbert space induced by $p$ if (cf. Ref. 10)

(ih1) $(\mathcal{K} ;\langle\cdot, \cdot\rangle)$ is a complex Hilbert space;

(ih2) $\Pi: \mathcal{V} \rightarrow \mathcal{K}$ is a linear operator with dense range;

(ih3) $p(u, v)=\langle\Pi u, \Pi v\rangle$ for all $u, v \in \mathcal{V}$.

The existence of an induced Hilbert space associated with any positive semidefinite Hermitian form follows by the well-known quotient-completion method: consider the factor space $\mathcal{V} / \operatorname{Ker}(p)$ onto which $p$ is positive definite and complete it to a Hilbert space $\mathcal{K}$. The canonical mapping $\Pi$ is the composition of the canonical surjection $\mathcal{V} \rightarrow \mathcal{V} / \operatorname{Ker}(p)$ with the embedding $\mathcal{V} / \operatorname{Ker}(p) \hookrightarrow \mathcal{K}$. In addition, the induced Hilbert space is unique, modulo a Hilbert space isomorphism, in the sense that if $\left(\mathcal{K}^{\prime} ; \Pi^{\prime}\right)$ is another Hilbert space induced by $p$, the linear mapping $U$, defined by $U \Pi u$ $=\Pi^{\prime} u$ for all $u \in \mathcal{V}$, extends uniquely to a unitary operator $U \in \mathcal{B}\left(\mathcal{K}, \mathcal{K}^{\prime}\right)$, such that $U \Pi=\Pi^{\prime}$.

Let $p$ and $q$ be two positive semidefinite Hermitian forms on $\mathcal{V}$. By definition, $q$ is dominated by $p$, denoted $p \leq q$, if $p(u, u) \leq q(u, u)$ for all $u \in \mathcal{V}$. This is the natural partial order on $Q(\mathcal{V})^{+}$, the set of all non-negative Hermitian forms on $\mathcal{V}$. Also, for any numbers $s, t \leq 0$ the mapping $s p$ $+t q: \mathcal{V} \times \mathcal{V} \rightarrow \mathrm{C}$ defined by $(s p+t q)(u, v)=s p(u, v)+t q(u, v)$ for all $u, v \in \mathcal{V}$ is a positive semidefinite Hermitian form on $\mathcal{V}$. Thus, $Q(\mathcal{V})^{+}$is a convex cone.

Fix $p \in Q(\mathcal{V})^{+}$and $\left(\mathcal{K}_{p} ; \Pi_{p}\right)$ its induced Hilbert space. If $q \leq p$ then, letting $\left(\mathcal{K}_{q} ; \Pi_{q}\right)$ be the Hilbert space induced by $q$, it follows that $\operatorname{Ker}(p) \subseteq \operatorname{Ker}(q)$ and that the linear mapping $J_{p, q}: \Pi_{p} \mathcal{V} \rightarrow \Pi_{q} \mathcal{V}$ defined by

$$
J_{p, q} \Pi_{p} u=\Pi_{q} u, \quad \text { for all } u \in \mathcal{V},
$$

is correctly defined and extends uniquely to a contraction $J_{p, q} \in \mathcal{B}\left(\mathcal{K}_{p}, \mathcal{K}_{q}\right)$. Thus, letting

$$
D_{p}(q)=J_{p, q}^{*} J_{p, q}
$$

we get a contraction $D_{p}(q) \in \mathcal{B}\left(\mathcal{K}_{p}\right)$, such that 


$$
q(u, v)=\left\langle D_{p}(q) \Pi_{p} u, \Pi_{p} v\right\rangle_{p} \text { for all } u, v \in \mathcal{V} .
$$

The condition (B6) uniquely determines the non-negative operator $D_{p}(q)$. It is now easy to see that the following holds.

Proposition 7.1: Let $p \in Q(\mathcal{V})^{+}$. The mapping $q \mapsto D_{p}(q)$ defined in (B5) and its inverse (B6) establish an affine and order-preserving isomorphism between the convex and partially ordered sets $\left(\left\{q \in Q(\mathcal{V})^{+} \mid q \leq p\right\} ; \leq\right)$ and $\left(\left\{A \in \mathcal{B}\left(\mathcal{K}_{p}\right) \mid 0 \leq A \leq I\right\} ; \leq\right)$.

The operator $D_{p}(q)$ is called the Radon-Nikodym derivative of the quadratic form $q$ with respect to $p$. Let us also observe that, similarly as Theorem 2.9, we get the following.

Lemma 7.2: Let $p, q \in Q(\mathcal{V})^{+}$be such that $q \leq p$, let $\left(\mathcal{K}_{p} ; \Pi_{p}\right)$ be the Hilbert space induced by $p$, and $D_{p}(q)$ the Radon-Nikodym derivative of $q$ with respect to $p$. Then

$$
\left(\mathcal{K}_{p} \ominus \operatorname{Ker}\left(D_{p}(q)\right) ; D_{p}(q)^{1 / 2} P_{\mathcal{K}_{p} \ominus \operatorname{Ker}\left(D_{p}(q)\right)} \Pi_{p}\right)
$$

is the Hilbert space induced by $q$.

Further, for $p, q \in Q(\mathcal{V})^{+}$, one says that $p$ uniformly dominates $q$, denoted $q \leq{ }_{u} p$, if for some $t>0$ we have $q \leq t p$. This is a partial preorder relation (reflexive and transitive) on $Q(\mathcal{V})^{+}$. It is immediate from Proposition 7.1 that we get the following.

Corollary 7.3: For any given $p \in Q(\mathcal{V})^{+}$, the mapping $q \mapsto D_{p}(q)$ defined in (B5) and its inverse (B6) establish an affine and order-preserving isomorphism between the partially ordered cones $\left(\left\{q \in Q(\mathcal{V})^{+} \mid q \leq_{\mathrm{u}} p\right\} ; \leq\right)$ and $\left(\mathcal{B}\left(\mathcal{K}_{p}\right)^{+} ; \leq\right)$.

The analog of the chain rule in Theorem 2.10 is the following.

Theorem 7.4: Let $p, q, r \in Q(\mathcal{V})^{+}$be such that $p \leq{ }_{u} q{ }_{u} r$. Then, modulo the identification of the Hilbert space induced by $q$ as in Lemma 7.2, we have

$$
D_{r}(p)=D_{r}(q)^{1 / 2} P_{\mathcal{K}_{r} \ominus \operatorname{Ker}\left(D_{r}(q)\right)} D_{q}(p) P_{\mathcal{K}_{r} \ominus \operatorname{Ker}\left(D_{r}(q)\right)} D_{r}(q)^{1 / 2} .
$$

\section{Closable non-negative quadratic forms}

Let $\mathcal{H}$ be a Hilbert space. In this subsection we consider the set $\mathcal{Q}(\mathcal{H})^{+}$, of all nonnegative (positive semidefinite) quadratic forms $p$, defined on some densely defined linear subspace $\operatorname{Dom}(p)$ of $\mathcal{H}$, more precisely, with the notation as in Appendix B 1,

$$
\mathcal{Q}(\mathcal{H})^{+}=\cup\left\{Q(\mathcal{V})^{+} \mid \mathcal{V} \text { dense in } \mathcal{H}\right\} .
$$

The natural partial order relation on $\mathcal{Q}(\mathcal{H})^{+}$is defined in the following way: given $p, q \in \mathcal{Q}(\mathcal{H})^{+}$ one says that $p$ dominates $q$, and we write $q \leq p$, if $\operatorname{Dom}(p) \subseteq \operatorname{Dom}(q)$ and $q(h, h) \leq p(h, h)$ for all $h \in \operatorname{Dom}(p)$. Another partial order is given by extensions: given $p, q \in \mathcal{Q}(\mathcal{H})$ one says that $p$ extends $q$, and we write $q \subseteq p$, if $\operatorname{Dom}(q) \subseteq \operatorname{Dom}(p)$ and $q(u, v)=p(u, v)$ for all $u, v \in \operatorname{Dom}(q)$.

A form $p \in \mathcal{Q}(\mathcal{H})^{+}$is called closed if any of the following equivalent conditions holds.

(i) $\operatorname{Dom}(p)$ endowed with the norm $\operatorname{Dom}(p) \ni h \mapsto(p(h)+\langle h, h\rangle)^{1 / 2}$ is a Hilbert space.

(ii) For any sequence $\left(h_{n}\right)$ of vectors in $\operatorname{Dom}(p)$ that converges in norm to $h \in \mathcal{H}$ and $p\left(h_{n}\right.$ $\left.-h_{m}\right) \rightarrow 0$ as $n, m \rightarrow \infty$, it follows that $h \in \operatorname{Dom}(p)$ and $p\left(h_{n}-h\right) \rightarrow 0$ as $n \rightarrow \infty$.

Further, $p$ is called closable if any of the following equivalent conditions holds (see Theorem VI.1.17 in Ref. 20).

(i) There exists a closed quadratic form $q$ that extends $p$.

(ii) For any sequence $\left(h_{n}\right)$ of vectors in $\operatorname{Dom}(p)$ that converges in norm to 0 and $p\left(h_{n}-h_{m}\right)$ $\rightarrow 0$ as $n, m \rightarrow \infty$, it follows that $p\left(h_{n}\right) \rightarrow 0$ as $n \rightarrow \infty$.

If $p$ is closable there exists the smallest closed extension of $p$, denoted by $\bar{p}$ and called the closure of $p$, which can be calculated in the following way: $\operatorname{Dom}(\bar{p})$ consists of all $h \in \mathcal{H}$ with the property that there exists a sequence $\left(h_{n}\right)$ of vectors in $\operatorname{Dom}(p)$ such that $\left\|h_{n}-h\right\| \rightarrow 0$ as $n \rightarrow \infty$ and $p\left(h_{n}-h_{m}, h_{n}-h_{m}\right) \rightarrow 0$ as $n, m \rightarrow \infty$, and $\bar{p}(h, h)=\lim p\left(h_{n}, h_{n}\right)$ for any such $h$ and $\left(h_{n}\right)$ as before. 
The importance of closed quadratic forms for spectral theory is partially justified by the following representation theorem (cf. Friedrichs, ${ }^{14}$ see Kato ${ }^{20}$ and Reed and Simon ${ }^{32}$ ).

Theorem 7.5: There exists a bijective correspondence $A \mapsto p_{A}$, between the set of all positive self-adjoint operators $A$ in $\mathcal{H}$ and the set of all closed densely defined positive semidefinite quadratic forms $p$ in $\mathcal{H}$, given by

$$
\operatorname{Dom}\left(p_{A}\right)=\operatorname{Dom}\left(A^{1 / 2}\right), \quad p_{A}(h, k)=\left\langle A^{1 / 2} h, A^{1 / 2} k\right\rangle, \quad h, k \in \operatorname{Dom}\left(A^{1 / 2}\right) .
$$

In the correspondence at (B8), the following assertions are equivalent.

(i) $\quad p_{B} \leq p_{A}$.

(ii) $\quad(A+I)^{-1} \leq(B+I)^{-1}$.

(See Theorem S.17 in Ref. 32.)

\section{Absolute continuity of non-negative quadratic forms}

Let $\mathcal{V}$ be a complex vector space. For $p, q \in Q(\mathcal{V})^{+}$, we say that $q$ is $p$-absolutely continuous, and we write $q \ll p$, if there exists a sequence $\left(q_{n}\right) \in Q(\mathcal{V})^{+}$subject to the following conditions.

(acf1) The sequence $\left(q_{n}\right)$ is nondecreasing, that is, $q_{n} \leq q_{n+1}$ for all $n \in \mathbb{N}$;

(acf2) $\lim _{n \rightarrow \infty} q_{n}(u, v)=q(u, v)$ for all $u, v \in \mathcal{V}$;

(acf3) $q_{n} \leq_{u} p$ for all $n \in \mathbb{N}$, that is, there exists a sequence $\left(t_{n}\right)$ of positive numbers such that $q_{n} \leq t_{n} p$ for all $n \in \mathbb{N}$.

The analog of Lemma 2.5 holds and then it is immediate from Proposition 7.1 the following.

Lemma 7.6: Let $p, q, r \in Q(\mathcal{V})^{+}$be such that $p, q \leq_{u}$. Then $q$ is $p$-absolutely continuous if and only if $D_{r}(q)$ is $D_{r}(p)$-absolutely continuous.

We are now in a position to show the connection of absolute continuity with closability of non-negative quadratic forms.

Theorem 7.7: Let $p, q \in Q(\mathcal{V})^{+}$and let $\left(\mathcal{K}_{p}, \Pi_{p}\right)$ be the Hilbert space induced by $p$. Then $q$ is $p$-absolutely continuous if and only if $\operatorname{Ker}(p) \subseteq \operatorname{Ker}(q)$ and the densely defined non-negative quadratic form $\tilde{q}$, with domain $\Pi_{p} \mathcal{V}$ in the Hilbert space $\mathcal{K}_{p}$ and defined by $\Pi_{p} v \mapsto q(v, v)$, is closable.

Proof: If $q$ is $p$-absolutely continuous, let the sequence $\left(q_{n}\right)$ in $Q(\mathcal{V})^{+}$have the properties (acf1)-(acf3), and it is easy to see from here that

$$
\operatorname{Ker}(p) \subseteq \cup_{n \geq 1} \operatorname{Ker}\left(q_{n}\right) \subseteq \operatorname{Ker}(q)
$$

Therefore, since $\operatorname{Ker}(p)=\operatorname{Ker}\left(\Pi_{p}\right)$, it follows that the non-negative quadratic form $\tilde{q}$, with domain $\Pi_{p} \mathcal{V}$ dense in the Hilbert space $\mathcal{K}_{p}$, is correctly defined by $\Pi_{p} v \mapsto q(v, v)$.

Let $r=p+q \in Q(\mathcal{V})^{+}$and consider its induced Hilbert space $\left(\mathcal{K}_{r} ; \Pi_{r}\right)$, as well as the RadonNikodym derivatives $D_{r}(p)$ and $D_{r}(q)$. Then

$$
D_{r}(p)+D_{r}(q)=I
$$

where $I$ denotes the identity operator on $\mathcal{K}_{r}$. Moreover, by Lemma 7.2 it follows that $\left(\mathcal{K}_{r} \ominus \operatorname{Ker}\left(D_{r}(p)\right) ; P_{\mathcal{K}_{r} \ominus \operatorname{Ker}\left(D_{r}(p)\right)} D_{r}(p)^{1 / 2} \Pi_{r}\right)$ is the Hilbert space induced by $p$. In order to simplify the notation, let us observe that, without loss of generality, we can assume that $D_{r}(p)$ is injective, and hence that, modulo a unitary equivalence, $\mathcal{K}_{p}=\mathcal{K}_{r}$ and $\Pi_{p}=D_{r}(p)^{1 / 2} \Pi_{r}$. Thus, with this assumption, the quadratic form $\tilde{q}$ has $\operatorname{Dom}(\widetilde{q})=D_{r}(p)^{1 / 2} \Pi_{r} \mathcal{V}$ dense in the Hilbert space $\mathcal{K}_{r}$ and

$$
\widetilde{q}\left(D_{r}(p)^{1 / 2} \Pi_{r} u, D_{r}(p)^{1 / 2} \Pi_{r} v\right)=\left\langle D_{r}(q)^{1 / 2} \Pi_{r} u, D_{r}(q)^{1 / 2} \Pi_{r} v\right\rangle_{r}, \quad u, v \in \mathcal{V} .
$$

Let us consider the operator $T$ densely defined in $\mathcal{K}_{r}$ by 


$$
T=D_{r}(q)^{1 / 2} D_{r}(p)^{-1 / 2}: \operatorname{Ran}\left(D_{r}(p)^{1 / 2}\right) \rightarrow \mathcal{K}_{r},
$$

and note that $\widetilde{q} \subseteq q_{T}$, where $q_{T}$ is the non-negative quadratic form represented by $T$,

$$
\operatorname{Dom}\left(q_{T}\right)=\operatorname{Dom}(T) \quad \text { and } \quad q_{T}(h, k)=\langle T u, T v\rangle_{r} \quad \text { for all } h, k \in \operatorname{Dom}(T) .
$$

Since $D_{r}(q)^{1 / 2}$ is bounded, it follows that the adjoint operator of $T$ is

$$
T^{*}=D_{r}(p)^{-1 / 2} D_{r}(q)^{1 / 2}, \quad \operatorname{Dom}\left(T^{*}\right)=\left\{h \in \mathcal{K}_{r} \mid D_{r}(q)^{1 / 2} h \in \operatorname{Ran}\left(D_{r}(p)^{1 / 2}\right)\right\},
$$

which, by Lemma 7.6 and Theorem 6.2, is dense in $\mathcal{K}_{r}$, and hence $T$ is closable. Therefore, its associated quadratic form $q_{T}$ is closable, so $\widetilde{q}$ is closable.

Conversely, assume that $\operatorname{Ker}(p) \subseteq \operatorname{Ker}(q)$ and that $\tilde{q}$ is closable. We make the construction of the operator $T$ as in (B11) and claim that it is closable. Basically, this is a consequence of the fact that $\Pi_{r}$ has dense range, but here are the details. Let $\left(h_{n}\right)$ be a sequence in $\operatorname{Dom}(T)$ $=\operatorname{Ran}\left(D_{r}(p)^{1 / 2}\right)$ such that

$$
h_{n}=D_{r}(p)^{1 / 2} k_{n} \rightarrow 0 \text { and } T h_{n}=D_{r}(q)^{1 / 2} k_{n} \rightarrow y \text { as } n \rightarrow \infty,
$$

and the convergences are to be understood in the norm $\|\cdot\|_{r}$ of $\mathcal{K}_{r}$, where $\left(k_{n}\right)$ is a sequence of vectors in $\mathcal{K}_{r}$. We have to prove that $y=0$. To see this, since the range of $\Pi_{r}$ is dense in $\mathcal{K}_{r}$, there exists a sequence $\left(u_{n}\right)$ of vectors in $\mathcal{V}$ such that $\left\|k_{n}-\Pi_{r} u_{n}\right\|_{r} \rightarrow 0$ as $n \rightarrow \infty$. Then, due to the continuity of $D_{r}(p)^{1 / 2}$ and $D_{r}(q)^{1 / 2}$ it follows that

$$
\lim _{n \rightarrow \infty}\left\|D_{r}(p)^{1 / 2} k_{n}-D_{r}(p)^{1 / 2} \Pi_{r} u_{n}\right\|_{r}=0=\lim _{n \rightarrow \infty}\left\|D_{r}(q)^{1 / 2} k_{n}-D_{r}(q)^{1 / 2} \Pi_{r} u_{n}\right\|_{r},
$$

and hence, by (B14) it follows that

$$
\lim _{n \rightarrow \infty}\left\|D_{r}(p)^{1 / 2} \Pi_{r} u_{n}\right\|_{r}=0, \quad \lim _{n, m \rightarrow \infty} \widetilde{q}\left(D_{r}(p)^{1 / 2} \Pi_{r}\left(u_{n}-u_{m}\right)\right)=0 .
$$

Taking into account of the second equality in (B15), these imply

$$
\|y\|_{r}=\lim _{n \rightarrow \infty}\left\|D_{r}(q)^{1 / 2} \Pi_{r} u_{n}\right\|_{r}^{2}=\lim _{n \rightarrow \infty}\left\langle D_{r}(q)^{1 / 2} \Pi_{r} u_{n}, D_{r}(q)^{1 / 2} \Pi_{r} u_{n}\right\rangle_{r}=\lim _{n \rightarrow \infty} \widetilde{q}\left(D_{r}(p)^{1 / 2} \Pi_{r} u_{n}\right)=0,
$$

where, at the last step, we used the assumption that $\widetilde{q}$ is closable. Thus, $y=0$ and hence $T$ is closable and, consequently, its adjoint $T^{*}$ is densely defined.

Finally, taking into account the formula for $\operatorname{Dom}\left(T^{*}\right)$ as in (B13), by Theorem 6.2 and Lemma 7.6 it follows that $q$ is $p$-absolutely continuous.

A form $q \in Q(\mathcal{V})^{+}$is called $p$-singular if $p \wedge q=0$, that is, the only $r \in Q(\mathcal{V})^{+}$satisfying $r$ $\leq p, q$ is the 0 quadratic form on $\mathcal{V}$.

Given $p, q \in Q(\mathcal{V})^{+}$, a $p$-Lebesgue decomposition of $q$ is a decomposition $q=q_{1}+q_{0}$, where $q_{0}, q_{1} \in Q(\mathcal{V})^{+}, q_{1}$ is $p$-absolutely continuous and $q_{0}$ is $p$-singular.

Theorem 7.8: Given any $p, q \in Q(\mathcal{V})^{+}$, there exists a $p$-Lebesgue decomposition of $q=q_{\mathrm{ac}}$ $+q_{s}$ such that $q_{\mathrm{ac}}$ is maximal among all forms $s \in Q(\mathcal{V})^{+}$that are p-absolutely continuous and $s$ $\leq q$.

The proof is perfectly similar with that of Theorem 3.1. We only write explicitly the constructions of $q_{\mathrm{ac}}$ and $q_{s}$, namely, for any $u, v \in \mathcal{V}$,

$$
q_{\mathrm{ac}}(u, v)=\left\langle D_{r}(q) P_{\mathcal{K}_{r} \ominus \operatorname{Ker}\left(D_{r}(p)\right)} \Pi_{r} u, \Pi_{r} v\right\rangle_{r}, \quad q_{s}(u, v)=\left\langle P_{\operatorname{Ker}\left(D_{r}(p)\right)} \Pi_{r} u, \Pi_{r} v\right\rangle_{r},
$$

where, $\left(\mathcal{K}_{r} ; \Pi_{r}\right)$ is the Hilbert space induced by the nonnegative quadratic form $r=p+q$.

If we combine Theorem 7.8 with Theorem 7.7 we get the following.

Corollary 7.9: [Simon (Ref. 33) Theorem 2.5] Let $p, q \in Q(\mathcal{V})^{+}$, let $\left(\mathcal{K}_{p} ; \Pi_{p}\right)$ be the Hilbert space induced by $p$ and $q=q_{\mathrm{ac}}+q_{s}$ the decomposition as in (B17). Then $q_{\mathrm{ac}}$ is maximal among all forms $s \in Q(\mathcal{V})^{+}$subject to the following conditions. 
(i) $s \leq q$.

(ii) $\operatorname{Ker}(s) \supseteq \operatorname{Ker}(p)$.

(iii) The non-negative quadratic form $\tilde{s}$, with $\operatorname{Dom}(\widetilde{s})=\Pi_{p} \mathcal{V}$ dense in the Hilbert space $\mathcal{K}_{p}$ and defined by $\Pi_{p} v \mapsto q(v, v)$, is closable.

In addition, in a similar fashion with Theorem 3.3 we get the following.

Theorem 7.10: Let $p, q, r \in Q(\mathcal{V})^{+}$be such that $r$ uniformly dominates $p$ and $q$. Let $q=q_{\mathrm{ac}}$ $+q_{s}$ be the $p$-Lebesgue decomposition of $q$ defined by (B17). Then, the Radon-Nikodym derivatives of $q_{\mathrm{ac}}$ and $q_{s}$, with respect to $r$, can be calculated as follows:

$$
D_{r}\left(q_{\mathrm{ac}}\right)=\left[D_{r}(p)\right] D_{r}(q) \text { and } D_{r}\left(q_{s}\right)=D_{r}(q)-\left[D_{r}(p)\right] D_{r}(q) \text {. }
$$

In particular, letting $\left(\mathcal{K}_{r} ; \Pi_{r}\right)$ be the Hilbert space induced by $r$, we have

$$
q_{\mathrm{ac}}(u, v)=\left\langle\left(\left[D_{r}(p)\right] D_{r}(q)\right) \Pi_{r} u, \Pi_{r} v\right\rangle_{r}, \quad u, v \in \mathcal{V},
$$

and

$$
q_{s}(u, v)=\left\langle\left(D_{r}(q)-\left[D_{r}(q)\right] D_{r}(q)\right) \Pi_{r} u, \Pi_{r} v\right\rangle, \quad u, v \in \mathcal{V} .
$$

The analog of Corollary 3.5 holds as well.

Corollary 7.11: Let $p, q \in Q(\mathcal{V})^{+}$. Then the following holds.

(i) $\quad q$ is $p$-absolutely continuous if and only if $q_{s}=0$.

(ii) $\quad q$ is $p$-singular if and only if $q_{\mathrm{ac}}=0$.

The transcription of Corollary 3.6 to the actual setting is clear as well.

Concerning the uniqueness of Lebesgue decompositions we have the following.

Theorem 7.12: Let $p \in Q(\mathcal{V})^{+}$be fixed.

(1) An arbitrary $q \in Q(\mathcal{V})^{+}$admits a unique $p$-Lebesgue decomposition if and only if $q_{\mathrm{ac}}$, the $p$-absolutely part of $q$, is uniformly dominated by $p$, that is, $q_{\mathrm{ac}} \leq t p$ for some $t>0$.

(2) The following assertions are equivalent.

(a) The property of $p$-absolute continuity is hereditary, that is, if $q, r \in Q(\mathcal{V})^{+}$are such that $r$ $\leq q \ll p$ then $r \ll p$.

(b) Any form $q \in Q(\mathcal{V})^{+}$admits a unique $p$-Lebesgue decomposition.

(c) The property of $p$-absolute continuity coincides with the property of p-uniform dominance, that is, for any $q \in Q(\mathcal{V})^{+}$, we have $q \ll p$ if and only if $q \leq{ }_{u} p$.

Proof:

(1) Let $r=p+q$. Since for any $p$-Lebesgue decomposition of $q=q_{1}+q_{0}$ we have $q_{1}, q_{2} \leq q \leq r$, it follows that the Radon-Nikodym derivative provides an order-preserving bijective correspondence between the set of all $p$-Lebesgue decompositions of $q$ and the set of all $D_{r}(p)$-Lebesgue decompositions of $D_{r}(q)$. Hence, the statement follows from Theorem 6.5.

(2) $\quad(a) \Rightarrow(b)$. This implication follows as in the proof of Proposition 3.8.

(b) $\Rightarrow$ (c). Let $q \in Q(\mathcal{V})^{+}$be such that $q \ll p$. Then, on the one hand $q=q_{\text {ac }}$ and, on the other hand, from (1) it follows that $q_{\mathrm{ac}} \leq_{u} p$, hence $q \leq{ }_{u} p$. Since $p$-uniform domination always implies $p$-absolute continuity, the proof is complete.

(c) $\Rightarrow$ (a). Clear.

An analog of Theorem 4.2 for the infimum of non-negative quadratic forms can be also obtained without any difficulty. 


\section{Radon-Nikodym derivatives for absolutely continuous non-negative quadratic forms}

We consider the same framework as before: $\mathcal{V}$ is a complex vector space and $Q(\mathcal{V})^{+}$denotes the set of non-negative quadratic forms on $\mathcal{V}$. In the following we use the notion of convergence in the strong resolvent sense (e.g., see Ref. 32): let $\left(A_{n}\right)$ be a sequence of (generally, unbounded) positive self-adjoint operators in a Hilbert space $\mathcal{H}$. One says that $\left(A_{n}\right)$ converges in the strong resolvent sense to the positive self-adjoint operator $A$ in $\mathcal{H}$ if SO- $\lim _{n \rightarrow \infty}\left(I+A_{n}\right)^{-1}=(I+A)^{-1}$. The name is justified by the known fact that, in this case, we also have SO- $\lim _{n \rightarrow \infty}\left(\zeta I-A_{n}\right)^{-1}=(\zeta I$ $-A)^{-1}$ for all $\zeta \in \mathrm{C} \backslash \mathbb{R}$ (e.g., see Corollary VIII.1.4 in Ref. 20). Also, given a closable operator $T$ in the Hilbert space, a subspace $\mathcal{D} \subseteq \operatorname{Dom}(T)$ is called a core for $T$ if the closure of $T \mid \mathcal{D}$ coincides with the closure of $T$

Theorem 7.13: Let $p, q \in Q(\mathcal{V})$ and let $\left(\mathcal{K}_{p} ; \Pi_{p}\right)$ be the Hilbert space induced by $p$.

(a) $q$ is p-absolutely continuous if and only if there exists a (generally unbounded) linear operator $D_{p}(q)$, uniquely determined by the following properties.

(i) $\quad D_{p}(q)$ is a positive self-adjoint operator in $\mathcal{K}_{p}$.

(ii) $\quad \Pi_{p} \mathcal{V}$ is a core for $D_{p}(q)^{1 / 2}$.

(iii) $\quad q(u, v)=\left\langle D_{p}(q)^{1 / 2} \Pi_{p} u, D_{p}(q)^{1 / 2} \Pi_{p} v\right\rangle_{p}$ for all $u, v \in \mathcal{V}$.

(b) Assume that $q$ is p-absolutely continuous. Then, for any sequence $\left(q_{n}\right)$ of quadratic forms in $Q(\mathcal{V})^{+}$that is nondecreasing, $q_{n}(u) \rightarrow q(u)$ for all $u \in \mathcal{V}$, and $q_{n} \leq_{u} p$ for all $n \in \mathbb{N}$, it follows that $D_{p}\left(q_{n}\right)$ converges to $D_{p}(q)$ in the strong resolvent sense.

Before approaching the proof of Theorem 7.13 we record a result of Simon, ${ }^{33}$ including its proof not only for the readers convenience but also as an illustration of the applicability of Lebesgue-type decompositions for non-negative quadratic forms. Recall that for two positive self-adjoint operators $A$ and $B$ acting in the same Hilbert space $\mathcal{H}$, one writes $A \leq B$ if $\operatorname{Dom}\left(B^{1 / 2}\right) \subseteq \operatorname{Dom}\left(A^{1 / 2}\right)$ and $\left\langle A^{1 / 2} h, A^{1 / 2} h\right\rangle \leq\left\langle B^{1 / 2} h, B^{1 / 2} h\right\rangle$ for all $h \in \operatorname{Dom}\left(B^{1 / 2}\right)$, that is, the natural order relation coming out from the order relation of the corresponding quadratic forms.

Lemma 7.14: [Simon (Ref. 33)] Let $\left(A_{n}\right)_{n \geq 1}$ be a sequence of (generally unbounded) positive self-adjoint operators in a Hilbert space $\mathcal{H}$ subject to the following properties.

(i) $\quad\left(A_{n}\right)_{n \geq 1}$ is nondecreasing in the associated quadratic forms order relation.

(ii) There exists a positive self-adjoint operator $A_{0}$ in $\mathcal{H}$ such that $A_{n} \leq A_{0}$ for all $n \geq 1$.

Then, letting

$$
\operatorname{Dom}(q)=\left\{h \in \underset{k \geq 1}{\cap} \operatorname{Dom}\left(A_{k}^{1 / 2}\right) \mid \sup _{n \geq 1}\left\|A_{n}^{1 / 2} h\right\|<\infty\right\}, \quad q(h)=\sup _{n \geq 1}\left\|A_{n}^{1 / 2} h\right\|^{2},
$$

$q$ is a closed non-negative quadratic form on $\mathcal{H}$ and letting $A$ denote the positive self-adjoint operator in $\mathcal{H}$ that represents the non-negative quadratic form $q$, the sequence $\left(A_{n}\right)$ converges in the strong resolvent sense to A.

Proof: Note that $\operatorname{Dom}(q) \supseteq \operatorname{Dom}\left(A_{0}^{1 / 2}\right)$ and hence it is dense in $\mathcal{H}$. We let $p=\|\cdot\|^{2}$ be the quadratic form associated with the scalar product on $\mathcal{H}$ and, for the moment, consider all the quadratic forms restricted to $\mathcal{V}=\operatorname{Dom}(q)$. For all $n \geq 1$ let $q_{n}=\left\|A_{n}^{1 / 2} \cdot\right\|^{2}$ denote the quadratic form corresponding to $A_{n}$ and restricted to $\mathcal{V}$ as well. Let $q=q_{\mathrm{ac}}+q_{s}$ be the $p$-Lebesgue decomposition of $q$ and observe, by the maximality property of $q_{\mathrm{ac}}$, that for all $n \geq 1$ we have $q_{n} \leq q_{\mathrm{ac}}$ because $q_{n}$ is closable. Therefore, $q \leq q_{\mathrm{ac}}$ hence $q=q_{\mathrm{ac}}$ is closable.

We claim that $q$ is closed. To see this, let $h \in \mathcal{H}$ and $\left(h_{n}\right)$ a sequence of vectors in $\operatorname{Dom}(q)$ such that $\left\|h_{n}-h\right\| \rightarrow 0$ and $q\left(h_{n}-h_{m}, h_{n}-h_{m}\right) \rightarrow 0$ as $m, n \rightarrow \infty$. Since for arbitrary $k \geq 1$ we have $q_{k} \leq q$ and $q_{k}$ is closable, it follows that $h \in \operatorname{Dom}\left(h_{k}\right)$, hence $h \in \cap_{k \geq 1} \operatorname{Dom}\left(h_{k}\right)$. On the other hand, by the triangle inequality for the seminorm $q^{1 / 2}$, 


$$
\left|q\left(h_{n}, h_{n}\right)^{1 / 2}-q\left(h_{m}, h_{m}\right)^{1 / 2}\right| \leq\left|q\left(h_{m}-h_{n}, h_{m}-h_{n}\right)\right| \text { for all } m, n \geq 1,
$$

hence $\sup _{n \geq 1} q\left(h_{n}, h_{n}\right)<\infty$. Then

$\sup _{n \in \mathbb{N}} q_{n}(h, h)=\sup _{n \in \mathbb{N}} \lim _{m \rightarrow \infty} q_{n}\left(h_{m}, h_{m}\right)=\sup _{n \in \mathbb{N}} \sup _{m \in \mathbb{N}} q_{n}\left(h_{m}, h_{m}\right)=\sup _{m \in \mathbb{N}} \sup _{n \in \mathbb{N}} q_{n}\left(h_{m}, h_{m}\right)=\sup _{m \in \mathbb{N}} q\left(h_{m}, h_{m}\right)<\infty$,

and hence $h \in \operatorname{Dom}(q)$, by definition. Now observe that the sequence $\left(h_{n}-h\right)$ is Cauchy in the seminorm $q^{1 / 2}$ and, since $\left\|h_{n}-h\right\| \rightarrow 0$ as $n \rightarrow \infty$, taking into account that $q$ is closable, it follows that $q\left(h_{n}-h, h_{n}-h\right) \rightarrow 0$ as $n \rightarrow \infty$. We conclude that $q$ is closed.

Finally, by Theorem 7.5 and hypotheses, the sequence of positive bounded operators $\left(A_{n}\right.$ $+I)^{-1}$ is nondecreasing and bounded from below by $\left(A_{0}+I\right)^{-1}$, hence it converges strongly to a positive bounded operator $B \geq\left(A_{0}+I\right)^{-1}$ and hence, there exists a unique positive self-adjoint operator $A$ in $\mathcal{H}$ such that $(A+I)^{-1}=B$. Clearly, the quadratic form $p_{A}$ corresponding to $A$ satisfies $q \leq p_{A}$. Since $p_{A_{n}} \leq q$ for all $n \geq 1$ it follows that $p_{A}=q$.

Proof of Theorem 7.13:

(a) Let us first assume that $q$ is $p$-absolutely continuous. By the first part of the proof of Theorem 7.7 it follows that, without loss of generality, we can assume that $\operatorname{Ker}\left(D_{r}(p)\right.$ $=\{0\}$ and then, letting $J_{p, q}$ denote the closure of the operator $T$ defined at (B11), we have [see (B13)]

$$
J_{p, q}^{*}=D_{r}(p)^{-1 / 2} D_{r}(q)^{1 / 2}, \quad \operatorname{Dom}\left(J_{p, q}^{*}\right)=\left\{h \in \mathcal{K}_{r} \mid D_{r}(q)^{1 / 2} \in \operatorname{Ran}\left(D_{r}(p)^{1 / 2}\right)\right\} .
$$

Thus, letting

$$
D_{p}(q)=J_{p, q}^{*} J_{p, q}
$$

by the celebrated theorem of von Neumann it follows that $D_{p}(q)$ is a positive selfadjoint operator in $\mathcal{K}_{p}$ and $\operatorname{Dom}\left(J_{p, q}\right)$ is a core of $D_{p}(q)$. On the other hand, with the notation as in the proof of Theorem 7.7, from (B9), and by Borelian functional calculus we get

$$
D_{p}(q)=D_{r}(p)^{-1 / 2} D_{r}(q) D_{r}(p)^{-1 / 2}, \quad \operatorname{Dom}\left(D_{p}(q)\right)=\operatorname{Ran}\left(D_{r}(p)^{1 / 2}\right),
$$

and that $\Pi_{p} \mathcal{V}$ is a core for $D_{p}(q)^{1 / 2}$. In addition, for arbitrary $u, v \in \mathcal{V}$ we have

$$
\begin{aligned}
& \left\langle D_{p}(q)^{1 / 2} \Pi_{p} u, D_{p}(q)^{1 / 2} \Pi_{p} v\right\rangle_{p} \\
& \quad=\left\langle D_{p}(q)^{1 / 2} D_{r}(p)^{1 / 2} \Pi_{r} u, D_{p}(q)^{1 / 2} D_{r}(p)^{1 / 2} \Pi_{r} v\right\rangle_{r} \\
& \quad=\left\langle J_{p, q} D_{r}(p)^{1 / 2} \Pi_{r} u, J_{p, q} D_{r}(p)^{1 / 2} \Pi_{r} v\right\rangle_{r}=\left\langle D_{r}(q)^{1 / 2} \Pi_{r} u, D_{r}(q)^{1 / 2} \Pi_{r} v\right\rangle_{r}=q(u, v) .
\end{aligned}
$$

The uniqueness part is clear.

(b) Let $\left(q_{n}\right)$ be a sequence in $Q(\mathcal{V})^{+}$as in the statement. Then the sequence of Radon-Nikodym derivatives $\left(D_{p}\left(q_{n}\right)\right)$ satisfies the assumptions in Lemma 7.14, with $A_{0}=D_{p}(q)$. It remains to observe that $h \in \mathcal{K}_{p}$ satisfies $\sup _{n \in \mathbb{N}}\left\|D_{p}\left(q_{n}\right)^{1 / 2} h\right\|<\infty$ if and only if $h \in \operatorname{Dom}\left(D_{p}(q)^{1 / 2}\right)$ and apply Lemma 7.14. 


\section{APPENDIX C: UNIFICATION OF THE THREE COMPARISON THEORIES}

\section{Recovering the maximal Lebesgue decomposition for non-negative operators}

So far we indicated how the maximal Lebesgue decomposition for completely positive maps (and, as shown in Appendix B, the whole comparison theory for nonnegative quadratic forms) can be derived from the comparison theory for non-negative bounded operators on a Hilbert space. Now we show that the converse way is also possible.

We first indicate the recovering of the maximal Lebesgue decomposition. If $\mathcal{A}$ is a $C^{*}$-subalgebra of $\mathcal{B}(\mathcal{H})$ and $A \in \mathcal{B}(\mathcal{H})^{+}$, we define the map

$$
\mu_{A}: \mathcal{A} \rightarrow \mathcal{B}(\mathcal{H}), \quad \text { by } \quad X \mapsto \mu_{A}(X)=A^{1 / 2} X A^{1 / 2} \text {. }
$$

It is easy to observe that $\mu_{A} \in \mathrm{CP}(\mathcal{A} ; \mathcal{H})$ and that, if $A \in \mathcal{A}^{\prime} \cap \mathcal{B}(\mathcal{H})$, then $\mu_{A}$ is the map of multiplication with $A$ (left is the same with right, in this case).

Theorem 8.1: Let $A, B \in \mathcal{B}(\mathcal{H})^{+}$and let $\mathcal{A}=C^{*}(I, A, B)^{\prime}$ be the commutant of the $C^{*}$-algebra generated by $I, A$, and $B$ in $\mathcal{B}(\mathcal{H})$. Let $\mu_{A}, \mu_{B}$, and $\mu_{I}$ be the completely positive maps defined as in (C1). Then $\mu_{I}$ uniformly dominates $\mu_{A}$ and $\mu_{B}$, and

$$
D_{\mu_{I}}\left(\mu_{A}\right)=A \text { and } D_{\mu_{I}}\left(\mu_{B}\right)=B .
$$

If $\mu_{B}=\mu_{B, \mathrm{ac}}+\mu_{B, s}$ is the maximal $\mu_{A}$-Lebesgue decomposition of $\mu_{B}$, then

$$
\mu_{B, \mathrm{ac}}=\mu_{[A] B} \text { and } \mu_{B, s}=\mu_{B-[A] B} .
$$

Proof: Note that $(\pi ; \mathcal{H} ; V)$, where $\pi: \mathcal{A} \hookrightarrow \mathcal{B}(\mathcal{H})$ is the canonical embedding and $V: \mathcal{H} \rightarrow \mathcal{H}$ is the identity operator, is the minimal Stinespring representation for $\mu_{I}$. Moreover,

$$
\mu_{A}=V^{*} A \pi(\cdot) V \quad \text { and } \quad \mu_{B}=V^{*} B \pi(\cdot) V .
$$

Since $0 \leq A, B \in \pi(\mathcal{A})^{\prime}$, they are the Radon-Nikodym derivatives of $\mu_{A}$ and, respectively, $\mu_{B}$ with respect to $\mu_{I}$. Since $\varphi_{\mathrm{ac}}=V^{*}[A] B \pi(\cdot) V$ and $\varphi_{s}=V^{*}(B-[A] B) \pi(\cdot) V$ the result follows.

Next we show how the whole comparison theory for non-negative operators can be recovered. Letting $\mathcal{H}$ be a Hilbert space we observe that there exists a bijective correspondence between $\mathrm{CP}(\mathrm{C} ; \mathcal{H})$ and $\mathcal{B}(\mathcal{H})^{+}$, given by $\mathrm{CP}(\mathrm{C} ; \mathcal{H}) \ni \varphi \mapsto \varphi(1) \in \mathcal{B}(\mathcal{H})^{+}$, with its inverse $\mathcal{B}(\mathcal{H})^{+} \ni A \mapsto \cdot A$ $\in \mathrm{CP}(\mathrm{C} ; \mathcal{H})$. It is easy to see that this mapping is affine and order preserving and hence a complete isomorphism.

\section{Recovering the comparison theory for non-negative bounded operators on Hilbert space}

Let $\mathcal{H}$ be a Hilbert space and consider the convex strict cone $Q(\mathcal{H})^{+}$of non-negative quadratic forms on $\mathcal{H}$, as in Appendix B 1. Let

$$
Q_{b}(\mathcal{H})^{+}=\left\{p \in Q(\mathcal{H})^{+} \mid p \text { is bounded }\right\},
$$

where the boundedness condition for $p$ means that $p(h) \leq C\|h\|^{2}$ for some $C>0$ and all $h \in \mathcal{H}$. The Riesz representation theorem shows that $p_{A}(h)=\langle A h, h\rangle, h \in \mathcal{H}$, establishes an affine and orderpreserving isomorphism between the convex strict cones,

$$
\mathcal{B}(\mathcal{H})^{+} \ni A \mapsto p_{A} \in Q_{b}(\mathcal{H})^{+} .
$$

On the other hand, since $\|\cdot\|^{2}=p_{I}$, where $I$ is the identity operator on $\mathcal{H}$, it follows that

$$
Q_{b}(\mathcal{H})^{+}=\left\{p \in Q(\mathcal{H}) \mid p \leq{ }_{u} p_{I}\right\}
$$

An inspection of the definitions of absolute continuity and Lebesgue decompositions shows that these notions are preserved by the above mentioned isomorphism between $Q_{b}(\mathcal{H})^{+}$and $\mathcal{B}(\mathcal{H})^{+}$. Thus, at least at the formal transcription of results, everything that can be proven in the compari- 
son theory of non-negative quadratic forms implies a corresponding result in the comparison theory of non-negative bounded operators on a Hilbert space. Moreover, the identification in (C3) can, alternatively, be viewed by means of the Radon-Nikodym derivative, that is, after observing that $(\mathcal{H} ; I)$ is the Hilbert space induced by $p_{I}$, it follows that in (C3) we actually have $A$ $=D_{p_{I}}\left(p_{A}\right)$.

${ }^{1}$ Anderson, W. N., Jr. and Duffin, R. J., "Series and parallel addition of matrices," J. Math. Anal. Appl. 26, 576 (1969).

${ }^{2}$ Anderson, Jr., W. N. and Trapp, G. E., "Shorted operators. II," SIAM J. Appl. Math. 28, 60 (1975).

${ }^{3}$ Ando, T., "Lebesgue-type decomposition of positive operators," Acta Sci. Math. 38, 253 (1976).

${ }^{4}$ Ando, T., Analytic and Geometric Inequalities and Applications, Math. Appllication (Kluwer Academic, Dordrecht, 1999), Vol. 478.

${ }^{5}$ Ando, T. and Szymański, W., "Order structure and Lebesgue decomposition of positive definite functions," Indiana Univ. Math. J. 35, 157 (1986).

${ }^{6}$ Arveson, W. B., "Subalgebras of $C^{*}$-algebras. I," Acta Math. 123, 141 (1969).

${ }^{7}$ Arveson, W. B., "Notes on extensions of $C^{*}$-algebras," Duke Math. J. 44, 329 (1977).

${ }^{8}$ Belavkin, V. P., D’Ariano, G. M., and Raginsky, M., "Operational distance and fidelity for quantum channels," J. Math. Phys. 46, 062106 (2005).

${ }^{9}$ Belavkin, V. P. and Staszewski, P., “A Radon-Nikodym theorem for completely positive maps," Rep. Math. Phys. 24, 49 (1986).

${ }^{10}$ Constantinescu, T. and Gheondea, A., "Representations of Hermitian kernels by means of Krein spaces," Publ. Res. Inst. Math. Sci. 33, 917 (1997).

${ }^{11}$ Choi, M.-D., "Completely positive linear maps on complex matrices," Linear Algebr. Appl. 10, 285 (1975).

${ }^{12}$ Davies, B. E., Quantum Theory of Open Systems (Academic, London, 1976).

${ }^{13}$ Fillmore, P. A. and Williams, J. P., "On operator ranges," Adv. Math. 7, 254 (1971).

${ }^{14}$ Friedrichs, K. O., "Spektraltheorie halbbeschränkter Operatoren und Anwendungen auf die Spektraltheorie von Differentzialoperatoren," Math. Ann. 109, 465 (1934).

${ }^{15}$ Gheondea, A., Gudder, S., and Jonas, P., "On the infimum of quantum effects," J. Math. Phys. 46, 062102 (2005).

${ }^{16}$ Gudder, S. P., “A Radon-Nykodim theorem of *-algebras," Pac. J. Math. 80, 141 (1979).

${ }^{17}$ Gudder, S. P., "Lattice properties of quantum effects," J. Math. Phys. 37, 2637 (1996).

${ }^{18}$ Hassi, S., Sebestyén, Z., de Snoo, H. S. V., and Szafraniec, F., "A canonical decomposition for linear operators and linear relations," Acta Math. Acad. Sci. Hung. 115, 281 (2007).

${ }^{19}$ Jørgensen, P. E. T., "Unbounded operators: perturbations and commutativity problems," J. Funct. Anal. 39, 281 (1980).

${ }^{20}$ Kato, T., Perturbation Theory for Linear Operators, 2nd ed. (Springer-Verlag, Berlin, 1976).

${ }^{21}$ Kosaki, H., "Remarks on Lebesgue-type decomposition of positive operators," J. Oper. Theory 11, 137 (1984).

${ }^{22}$ Kosaki, H., "Lebesgue decomposition of states in a von Neumann algebra," Am. J. Math. 107, 697 (1985).

${ }^{23}$ Kraus, K., "General state changes in quantum theory," Ann. Phys. 64, 311 (1971).

${ }^{24}$ Kraus, K., States, Effects, and Operations (Springer-Verlag, Berlin, 1983).

${ }^{25}$ Leung, D., "Choi's proof as a recipe for quantum tomography," J. Math. Phys. 44, 528 (2003).

${ }^{26}$ Meyer, P. A., Quantum Probability for Probabilists (Springer-Verlag, Berlin, 1993).

${ }^{27}$ Parthasarathy, K. R., Introduction to Quantum Stochastic Calculus (Birkhäuser Verlag, Basel, 1992).

${ }^{28}$ Parthasarathy, K. R., "Comparison of completely positive maps on a $C^{*}$-algebra and a Lebesgue decomposition theorem," in Athens Conference on Applied Probability and Time Series Analysis I, Lecture Series in Statistics Vol. 114 (Springer-Verlag, Berlin, 1996), pp. 34-54.

${ }^{29}$ Paulsen, V., Completely Bounded Maps and Operator Algebras (Cambridge University Press, Cambridge, 2002).

${ }^{30}$ Pekarev, E. L. and Shmulyan, Yu. L., "Parallel addition and parallel subtraction of operators," Izv. Akad. Nauk SSSR, Ser. Mat. 40, 366 (1976).

${ }^{31}$ Raginsky, M., "Radon-Nikodym derivatives of quantum operations," J. Math. Phys. 44, 5003 (2003).

${ }^{32}$ Reed, M. and Simon, B., Methods in Modern Mathematical Physics I. Functional Analysis (Revised and Enlarged Edition) (Academic, New York, 1980).

${ }^{33}$ Simon, B., "A canonical decomposition for quadratic forms with applications to monotone convergence theorems," J. Funct. Anal. 28, 377 (1978).

${ }^{34}$ Stinespring, W. F., "Positive functions on $C^{*}$-algebras," Proc. Am. Math. Soc. 6, 211 (1955). 